University of South Carolina

Scholar Commons

1998

\title{
Evaluation of the Acoustic Doppler Velocimeter (ADV) for Turbulence Measurements
}

George Voulgaris

University of South Carolina - Columbia, gvoulgaris@geol.sc.edu

John H. Trowbridge

Follow this and additional works at: https://scholarcommons.sc.edu/geol_facpub

Part of the Earth Sciences Commons

\section{Publication Info}

Published in Journal of Atmospheric and Oceanic Technology, Volume 15, Issue 1, 1998, pages 272-289. Voulgaris, G. \& Trowbridge, J. H. (1998). Evaluation of the acoustic doppler velocimeter (ADV) for turbulence measurements. Journal of Atmospheric and Oceanic Technology, 15 (1), 272-289.

(c) Copyright 1998 AMS

This Article is brought to you by the Earth, Ocean and Environment, School of the at Scholar Commons. It has been accepted for inclusion in Faculty Publications by an authorized administrator of Scholar Commons. For more information, please contact digres@mailbox.sc.edu. 


\title{
Evaluation of the Acoustic Doppler Velocimeter (ADV) for Turbulence Measurements*
}

\author{
G. Voulgaris AND J. H. Trowbridge \\ Woods Hole Oceanographic Institution, Woods Hole, Massachusetts
}

(Manuscript received 31 July 1996, in final form 26 March 1997)

\begin{abstract}
Accuracy of the acoustic Doppler velocimeter (ADV) is evaluated in this paper. Simultaneous measurements of open-channel flow were undertaken in a 17-m flume using an ADV and a laser Doppler velocimeter. Flow velocity records obtained by both instruments are used for estimating the true ("ground truth") flow characteristics and the noise variances encountered during the experimental runs. The measured values are compared with estimates of the true flow characteristics and values of variance $\left(\left\langle u^{\prime 2}\right\rangle,\left\langle w^{\prime 2}\right\rangle\right)$ and covariance $\left(\left\langle u^{\prime} w^{\prime}\right\rangle\right)$ predicted by semiempirical models for open-channel flow. The analysis showed that the ADV sensor can measure mean velocity and Reynolds stress within $1 \%$ of the estimated true value. Mean velocities can be obtained at distances less than $1 \mathrm{~cm}$ from the boundary, whereas Reynolds stress values obtained at elevations greater than $3 \mathrm{~cm}$ above the bottom exhibit a variation that is in agreement with the predictions of the semiempirical models. Closer to the boundary, the measured Reynolds stresses deviate from those predicted by the model, probably due to the size of the ADV sample volume. Turbulence spectra computed using the ADV records agree with theoretical spectra after corrections are applied for the spatial averaging due to the size of the sample volume and a noise floor. The noise variance in ADV velocity records consists of two terms. One is related to the electronic circuitry of the sensor and its ability to resolve phase differences, whereas the second is flow related. The latter noise component dominates at rapid flows. The error in flow measurements due to the former noise term depends on sensor velocity range setting and ranges from \pm 0.95 to $\pm 3.0 \mathrm{~mm} \mathrm{~s}^{-1}$. Noise due to shear within the sample volume and to Doppler broadening is primarily a function of the turbulence dissipation parameter. Noise variances calculated using spectral analysis and the results of the ground truthing technique are compared with theoretical estimates of noise.
\end{abstract}

\section{Introduction}

Boundary layer dynamics in the presence of winddriven flows, waves, and tides are complicated phenomena dominated by rapid momentum fluxes through intermittent, convective processes. Experimental study of these processes requires flow-measuring devices with adequate spatial and temporal resolution.

During the past 20 years, extensive experimental research has been undertaken on the mean and turbulence characteristics of open channel flow with the aid of hotfilm anemometers (e.g., Blinco and Partheniades 1971; Nakagawa and Nezu 1981) and various visualization methods such as the hydrogen-bubble technique (Grass 1971). In addition, the introduction of the laser Doppler velocimetry (LDV) provided another tool for nonintru-

\footnotetext{
* Woods Hole Oceanographic Institution Contribution Number 9300 .

Corresponding author address: George Voulgaris, Dept. of Geology and Geophysics, Woods Hole Oceanographic Institution, Mail Stop 22, Woods Hole, MA 02543.

E-mail: gvoulgaris@whoi.edu
}

sive measurements of turbulent flow in the laboratory (e.g., Nezu and Rodi 1986).

Similarly, field turbulence and sediment transport process studies require the use of rapidly responding flowmeasuring devices. Electromagnetic current meters are used widely for flow measurements in environments varying from the benthic boundary layer (e.g., Cacchione and Drake 1979; Voulgaris et al. 1995) to the surf zone (e.g., Guza and Thornton 1980). Although these sensors are robust enough to withstand the extremely hostile conditions of coastal seas, they suffer from zerodrift problems and they disturb the flow. Also, they are able to measure only two components of the flow, whereas their relatively large size (varying from 5 to $11 \mathrm{~cm}$ ) and slow response make them inappropriate for resolving fine scales of turbulence.

The development of travel-time acoustic sensors (e.g., Williams et al. 1987) offered simultaneous measurements of the instantaneous three-dimensional flow components at high sampling rates. The sensors require no calibration and have low noise levels, but their large sample volume limits the resolution of turbulence eddy scales and the proximity to the boundary.

Current in situ research on turbulence requires laboratory-quality measurements to be obtained in the field. The use of laboratory instrumentation in the field suffers 


\section{ADV vs LDV}
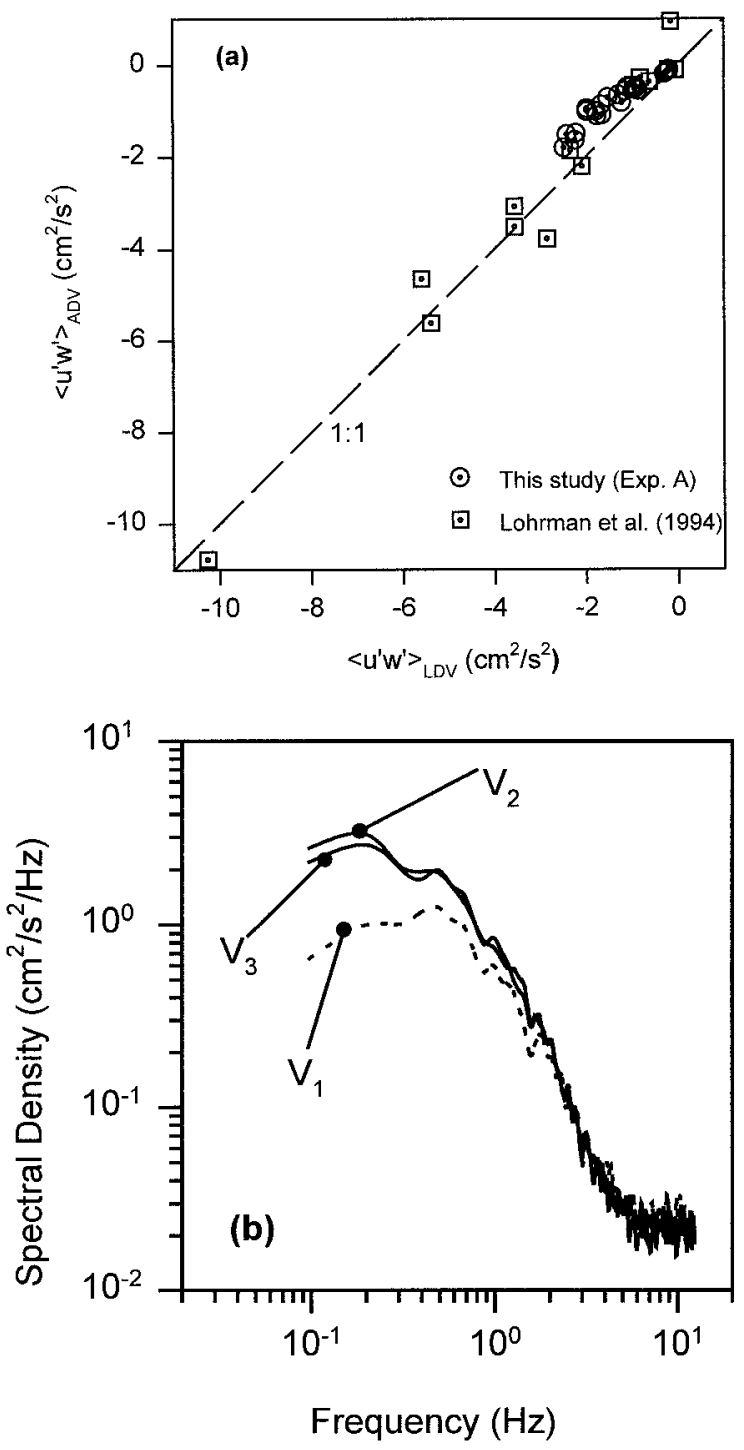

from problems varying from sensor calibration and corrosion problems (as in hot-wire anemometry) to deterioration of optical transmissions under high turbidity levels (as in laser systems; see Agrawal and Aubrey 1992; Trowbridge and Agrawal 1995) and high costs.

The acoustic Doppler velocimeter (ADV) is a new acoustic instrument with the potential to offer accuracy of laboratory devices even under field conditions (Lohrmann et al. 1994). It offers unobstructed three-dimensional flow measurements at high sampling rates and with a small sample volume. Field deployments have demonstrated that the sensor can be easily deployed either as a moored instrument (Anderson and Lohrmann 1995) or attached to a still structure near the seabed (Lohrmann et al. 1995). Lohrmann et al. (1995) reported
FIG. 1. (a) Direct intercomparison of Reynold stress values derived from collocated ADV and LDV measurements in a flume. Squares represent data collected by Lohrmann et al. (1994) in experiments with mean velocities varying from 5 to $80 \mathrm{~cm} \mathrm{~s}^{-1}$, whereas circles denote data collected in the present study and with mean velocities ranging from 5 to $35 \mathrm{~cm} \mathrm{~s}^{-1}$. The straight line depicts a 1:1 relationship between the LDV and the ADV values. (b) Spectral density of the three velocity components $\left(V_{1}, V_{2}, V_{3}\right)$ along the bisector of the transmit and receive axes showing that the noise level (spectral density at high frequencies) is of similar magnitude. (c) Cospectrum of the same velocity components as in (b) showing that the noise variance along the three acoustic beams is uncorrelated.

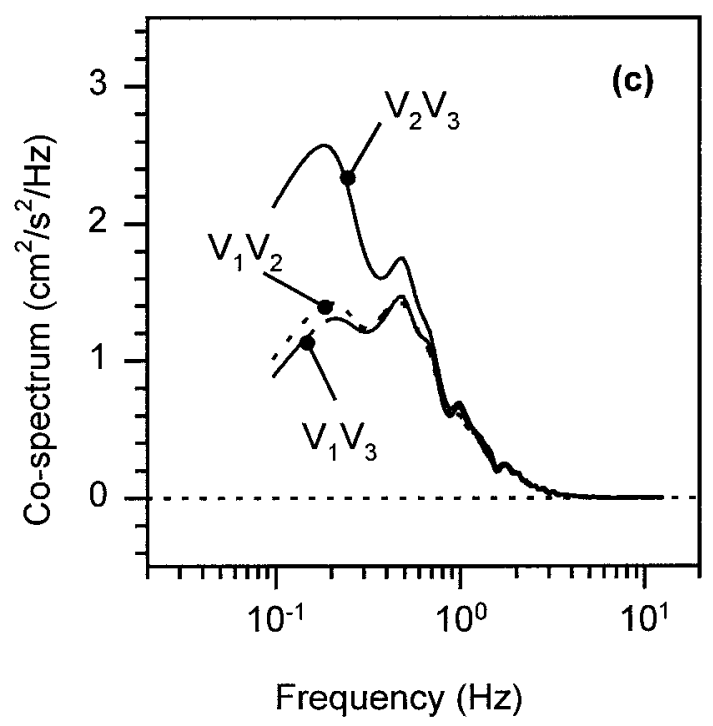

that during field deployment the sensor managed to withstand forces induced by 3-m breaking waves.

Earlier investigations of the accuracy of the ADV sensor (Lohrmann et al. 1994) concentrated on simply comparing the ADV sensor with measurements obtained using an LDV sensor. The results of this work were qualitative and showed a good agreement between the mean values obtained by the two sensors. Reynolds stresses were overestimated by the ADV at low shear stresses, whereas good agreement was stated at extremely high stresses (Fig. 1a). These results, however, are not conclusive and are indicative of the degree of agreement or disagreement of the two systems; they do not have of an objective method for the evaluation of any of the sensors. 
In the present work, the ability of the ADV sensor to measure turbulence is examined rigorously by comparing measured turbulence parameters to estimates of true flow values and turbulence spectra models. Combined analysis of the ADV and LDV data, which are two independent measurements, is used for the estimation of the true flow characteristics $\left(\langle u\rangle,\left\langle u^{\prime 2}\right\rangle,\left\langle w^{\prime 2}\right\rangle\right.$, and $\left.\left\langle u^{\prime} w^{\prime}\right\rangle\right)$ and the separation of the signal and noise components for each instrument. Measurements close to the bottom allow examination of the effect of proximity to the boundary. In addition, noise variances are obtained from spectral analysis. The noise level encountered in the ADV measurements is analyzed in its constituent components and compared to existing theoretical estimations of noise due to Doppler broadening (e.g., Cabrera et al. 1987) and shear of the flow within the sample volume.

In summary, the work presented here examines 1) the degree of accuracy in measuring mean velocity, variance, and covariance of the flow field; 2) the effect of the proximity to the boundary in flow measurements; and 3 ) the types and magnitudes of noise involved in flow measurements using an ADV sensor.

\section{Acoustic Doppler velocimeter}

\section{a. System description}

The ADV is a remote-sensing, three-dimensional velocity sensor, originally developed and tested for use in physical model facilities (Kraus et al. 1994). Its operation is based on the Doppler shift effect. It is implemented as a bistatic (focal point) acoustic Doppler system and consists of a transmitter and three receivers. The three $10-\mathrm{MHz}$ receivers are positioned in $120^{\circ}$ increments on a circle around a $10-\mathrm{MHz}$ transmitter. The probe is submerged in the flow and the receivers are slanted at $30^{\circ}$ from the axis of the transmit transducer, focusing on a common sample volume that is located approximately $10.8 \mathrm{~cm}$ from the probe, which ensures nonintrusive flow measurements (Fig. 2a). The size of the sample volume is a function of the length of the transmit pulse $(4.8 \mu$ s, i.e., $7.2 \mathrm{~mm})$, the width of the receive window, and the beam pattern of the receive and transmit pulses. The first parameter defines the vertical extent of the sample volume, whereas the latter two parameters define its lateral extent. Lohrmann et al. (1994) noted that the shape of the horizontal extent of the sample volume is slightly asymmetric toward the receive transducer. However, their experiments showed that this asymmetry is not significant and the lateral dimension $\left(d_{u}\right)$ can be calculated by the transducer diameter $(6 \mathrm{~mm})$ and the half-power beamwidth $\left(1.4^{\circ}\right)$. Here, $d_{u}$ is calculated to be $6.53 \mathrm{~mm}$. In a typical configuration of the ADV, the receive window is longer than the transmit pulse, resulting in a vertical scale $\left(d_{w}\right)$ for the sample volume of $9 \mathrm{~mm}$.

The system operates by transmitting short acoustic pulses along the transmit beam. As the pulses propagate through the water column, a fraction of the acoustic energy is scattered back by small particles suspended in the water (Fig. 2a). The phase data $(d \phi / d t)$ from successive coherent acoustic returns are converted into velocity estimates using a pulse-pair processing technique (Miller and Rochwarger 1972). The phase data are then converted into speed using the Doppler relation

$$
U=\frac{c(d \phi / d t)}{4 \pi f},
$$

where $f$ is the ADV's operating frequency (10 MHz) and $c$ the speed of sound in water. The phase data are given by

$$
\frac{d \phi}{d t}=\frac{1}{\tau} \tan ^{-1}\left[\frac{s(t) c(t+\tau)-s(t+\tau) c(t)}{c(t) c(t+\tau)+s(t) s(t+\tau)}\right],
$$

where $\phi$ is the signal phase in radians; $t$ is time; $\tau$ is the time between transmissions; and $c(t)$ and $s(t)$ are $\cos [\phi(t)]$ and $\sin [\phi(t)]$, respectively, so that the received signal can be written as a complex number $c(t)+$ $\sqrt{-1} s(t)$. An 8-bit A/D converter is used to capture the real and imaginary components of the signal. The cross and autocorrelations required in Eq. (2) are computed by the ADV for successive pulse pairs and are averaged over a number of transmissions before computing the flow speed. The time between transmissions $(\tau)$, the number of pulses averaged $(M)$, and the maximum velocities that can be measured are interrelated and are set by the user through the velocity range configuration. The option of five different nominal velocity ranges $\left( \pm 3, \pm 10, \pm 30, \pm 100\right.$, and $\left.\pm 250 \mathrm{~cm} \mathrm{~s}^{-1}\right)$ is given to the user. The actual velocity range along the bistatic angle (beam velocity range) and the pulse characteristics corresponding to each range are listed in Table 1. The system offers the advantage of being inherently drift free; it requires no routine calibration; and acoustic pulses do not suffer the range of limitations of optical pulses in turbid water, which is common particularly near the seabed.

The three current speed components measured along the bistatic angle directions, using Eqs. (1) and (2), are converted into the orthogonal coordinate system utilizing a transformation matrix $\mathbf{T}$ :

$$
\left[\begin{array}{c}
\mathrm{u} \\
\mathrm{v} \\
\mathrm{w}
\end{array}\right]=\mathbf{T} \cdot\left[\begin{array}{c}
V_{1} \\
V_{2} \\
V_{3}
\end{array}\right],
$$

where $(\mathrm{u}, \mathrm{v}, \mathrm{w})$ is the velocity vector in the orthogonal coordinate system and $V_{1}, V_{2}$, and $V_{3}$ are the velocity components along each acoustic axis (i.e., along the bisector of the transmit and receive axes, hereafter also called beam or acoustic beam). The values of the elements of the transformation matrix depend on the relative position of the three receivers and the transmitter. They are determined empirically by calibrating the 


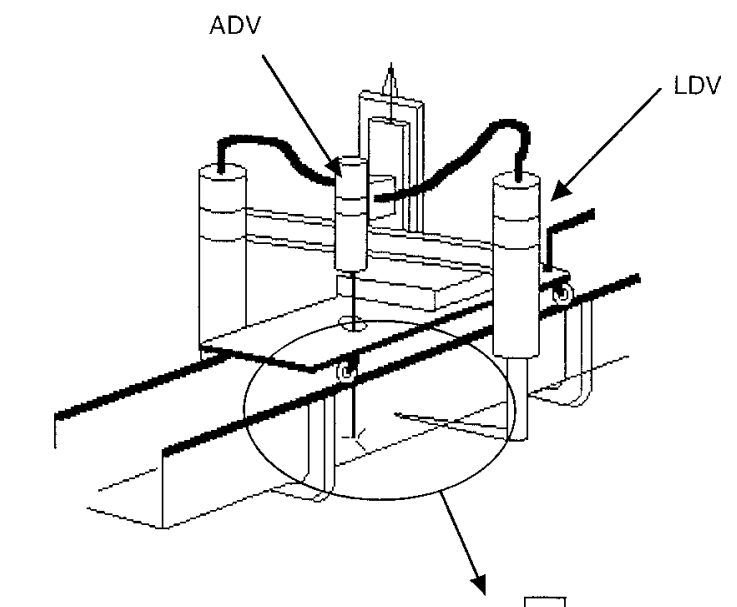

(b)

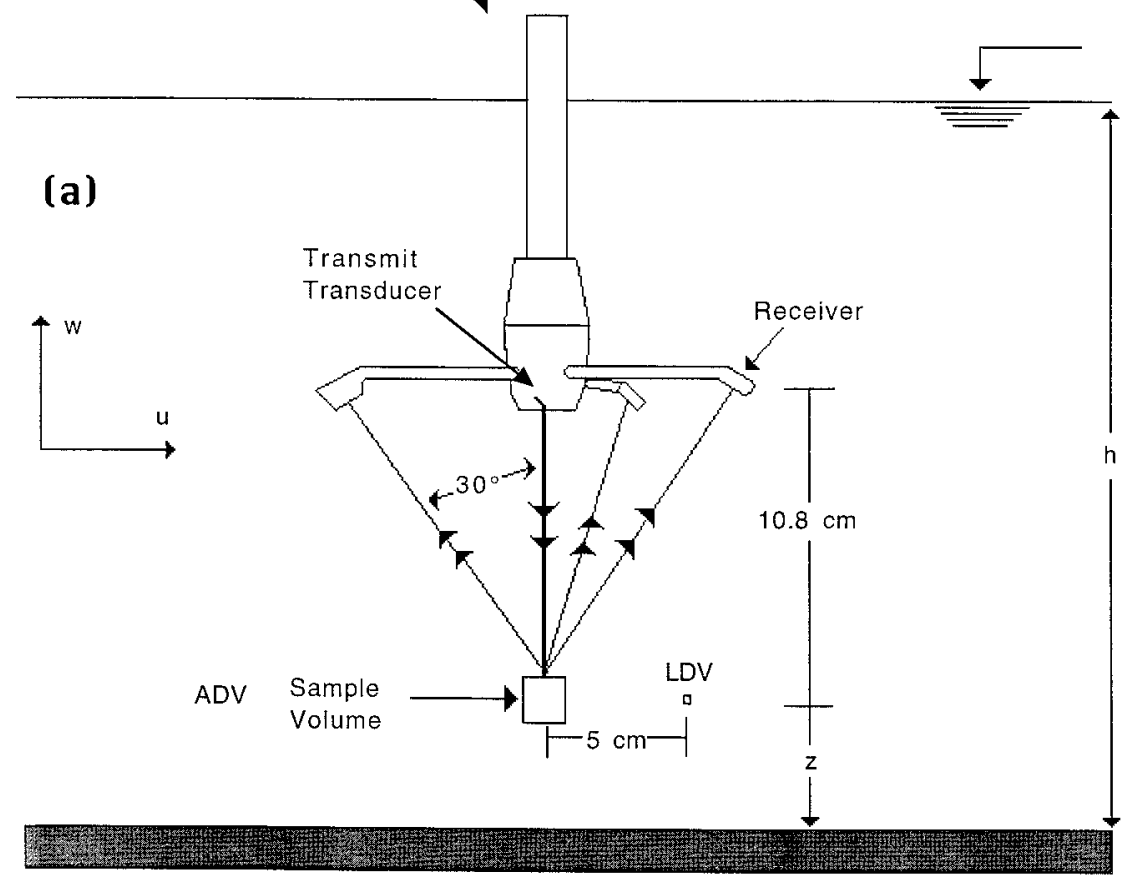

FIG. 2. Schematic diagram showing (a) the ADV transmit transducer/receiver layout and the principle of operation and (b) installation configuration during data collection.

TABLE 1. List of ADV velocity ranges (nominal and actual), time between pulse transmissions $(\tau)$, and number of pulses $(M)$ used for one velocity estimate. The variance of the velocity due to electronics $\left(\sigma_{m}^{2}\right)$ was determined experimentally in still water measurements, whereas the phase variance $\left(\sigma_{s}^{2}\right)$ has been estimated using Eq. (10) with $k=1.4$. Finally, the standard error $\left(\sigma_{s}\right)$ in measuring flow velocity along the beam direction and for different velocity range settings is shown in the last column.

\begin{tabular}{|c|c|c|c|c|c|c|}
\hline $\begin{array}{c}\text { Nominal velocity } \\
\text { range } \\
\left(\mathrm{cm} \mathrm{s}^{-1}\right)\end{array}$ & $\begin{array}{c}\text { Beam velocity } \\
\text { range } \\
\left(\mathrm{cm} \mathrm{s}^{-1}\right)\end{array}$ & $\begin{array}{c}\tau \\
(\mathrm{ms})\end{array}$ & $M$ & $\begin{array}{c}\sigma_{m}^{2} \\
\left(\mathrm{~cm}^{2} \mathrm{~s}^{-2}\right)\end{array}$ & $\sigma_{s}^{2}$ & $\begin{array}{c}\text { Beam velocity } \\
\text { error } \\
\left(\mathrm{mm} \mathrm{s}^{-1}\right)\end{array}$ \\
\hline \pm 3 & \pm 9.4 & 7.14 & 5 & $9.1 \times 10^{-3}$ & 0.83 & \pm 0.95 \\
\hline \pm 10 & \pm 15.6 & 5.55 & 6 & $9.5 \times 10^{-3}$ & 0.63 & \pm 0.97 \\
\hline \pm 30 & \pm 33.0 & 4.35 & 8 & $2.0 \times 10^{-2}$ & 1.08 & \pm 1.41 \\
\hline \pm 100 & \pm 78.0 & 4.00 & 9 & $1.1 \times 10^{-1}$ & 5.91 & \pm 3.00 \\
\hline \pm 250 & \pm 94.0 & 3.85 & 9 & $7.9 \times 10^{-2}$ & 3.78 & \pm 2.80 \\
\hline
\end{tabular}


transmitter-receiver system at a constant speed and for various angles of attack (Lohrmann et al. 1994). The transformation matrix is unique for each sensor, and the value of the matrix remains unchanged unless physical damage occurs to the sensor. For the sensor used in this study (SonTek probe number: 1192), the transformation matrix provided by the manufacturer was

$$
\mathbf{T}=\left[\begin{array}{lll}
a_{11} & a_{12} & a_{13} \\
a_{21} & a_{22} & a_{23} \\
a_{31} & a_{32} & a_{33}
\end{array}\right]=\left[\begin{array}{rrr}
2.73 & -1.40 & -1.33 \\
-0.04 & 2.33 & -2.29 \\
0.35 & 0.35 & 0.33
\end{array}\right] .
$$

Assuming that the measured velocity along each bistatic angle (beam) consists of the true velocity plus unbiased noise and that the noise in individual channels (beams) have identical variances but are uncorrelated, then it has been shown (Lohrmann et al. 1995) that measured values of turbulence characteristics deviate from the true flow values (denoted with a tilde) by a quantity that depends on the noise part multiplied by a factor

$$
\begin{aligned}
\left\langle u^{\prime} w^{\prime}\right\rangle & =\left\langle\tilde{u}^{\prime} \tilde{w}^{\prime}\right\rangle+\left(a_{11} a_{31}+a_{12} a_{32}+a_{13} a_{33}\right) \sigma_{t}^{2}, \\
\left\langle u^{\prime 2}\right\rangle & =\left\langle\tilde{u}^{\prime 2}\right\rangle+\left(a_{11}^{2}+a_{12}^{2}+a_{13}^{2}\right) \sigma_{t}^{2}, \\
\left\langle v^{\prime 2}\right\rangle & =\left\langle\tilde{v}^{\prime 2}\right\rangle+\left(a_{21}^{2}+a_{22}^{2}+a_{23}^{2}\right) \sigma_{t}^{2},
\end{aligned}
$$

and

$$
\left\langle w^{\prime 2}\right\rangle=\left\langle\tilde{w}^{\prime 2}\right\rangle+\left(a_{31}^{2}+a_{32}^{2}+a_{33}^{2}\right) \sigma_{t}^{2},
$$

where $\sigma_{t}^{2}$ is the variance of the noise $\left(\left\langle V_{n 1}^{2}\right\rangle=\left\langle V_{n 2}^{2}\right\rangle=\right.$ $\left.\left\langle V_{n 3}^{2}\right\rangle\right)$; the prime denotes fluctuating part of the velocity (i.e., $u^{\prime}=u-\langle u\rangle$ and $w^{\prime}=w-\langle w\rangle$ ). Equations (5)(8) show that the quality of the variance and covariance terms of the measured velocity depends on the noise level and the values of the transformation matrix (i.e., geometry of the sensor). For an ideally constructed sensor, the parameter $a_{11} a_{31}+a_{12} a_{32}+a_{13} a_{31}$ equals zero. In reality, though, this is very difficult to achieve. For example, for the sensor used in the present study, this parameter was 0.0266. This shows that the Reynolds stress measurements are not affected significantly by the noise level. However, the noise part of the variances is $10.67,11.18$, and 0.35 times the noise level along the bistatic angle measuring beam for the $u$, $v$, and $w$ components, respectively. At this point, it should be noted that if the noise variances among the three acoustic beams are not equal in magnitude, then this would result in an increased error in the estimation of the flow variances and covariances. However, such imbalances in noise levels signify a problem with the operation of the sensor that should be rectified prior to any measurement program.

Besides the noise along the transmit beam, the variance and covariance of flow measured by the ADV will be affected by the size of the sample volume. George and Lumley (1973) examined the effect of the size of sample volume to flow measurements using an LDV. Using the same approach, the ADV-measured variance spectra of the flow will be attenuated according to the transfer function with squared amplitudes given by $W \mathbf{k}$ $=\exp \left[-0.25\left(k_{u}^{2} d_{u}^{2}+k_{v}^{2} d_{v}^{2}+k_{w}^{2} d_{w}^{2}\right)\right]$, where $\mathbf{k}=\left(k_{u}, k_{v}\right.$, $\left.k_{w}\right)$ are the turbulence spectra wavenumbers and $\left(d_{u}, d_{v}\right.$, $\left.d_{w}\right)$ are the sample volume lengths along the $(x, y, z)$ directions. Following George and Lumbey's analysis [1973, 331, Eq. (3.1.23)] it can be shown that the attenuation of the one-dimensional energy level measured by the ADV depends on the turbulence microscale. In flows with high Reynolds numbers, the downstream and transverse turbulent spectra measured by the ADV sensor are attenuated by $50 \%$ at one-dimensional wavenumbers $\left(k_{1}\right)$ that correspond to eddy lengths of approximately 6 and $3 \mathrm{~cm}$, respectively.

\section{b. Sources of noise}

The total velocity error variance $\left(\sigma_{t}^{2}\right)$ along the transmit beam is the sum of 1) sampling errors $\left(\sigma_{m}^{2}\right)$ due to the ability of the system to resolve the phase shift $(d \phi /$ $d t$ ) of the return pulse; 2 ) errors due to random scatterer motions within the sample volume (Doppler noise, $\sigma_{D}^{2}$ ); and 3 ) errors due to mean velocity shear $\left(\sigma_{u}^{2}\right)$ within the sample volume (Lhermitte and Lemmin 1994), so that

$$
\sigma_{t}^{2}=\sigma_{m}^{2}+\sigma_{D}^{2}+\sigma_{u}^{2}
$$

The values measured by the ADV circuitry are voltages proportional to $\sin \phi(t)$ and $\cos \phi(t)$; thus, the accuracy of the ADV is ultimately limited by the ability of the electronics and the A/D converter to resolve the phase. For a coherent sensor like the ADV, the flow velocity estimated by averaging a number of acoustic pulse pairs, of duration $\tau$, will have an uncertainty (Zedel et al. 1996)

$$
\sigma_{m}^{2}=\frac{c^{2}}{4} \frac{1}{f^{2}} \frac{1}{4 \pi^{2}} K^{2} \sigma_{s}^{2} \frac{1}{\tau} \frac{1}{\left(T-t_{o}\right)},
$$

where $T$ is the time between successive estimates of one velocity value ( $1 / T$ equals sensor's sampling frequency); $t_{o}$ is the overhead time $(2 \mathrm{~ms})$ required by the sensor to carry out the necessary conversions; $K$ is an empirical constant (1.4, Zendel et al. 1996), while $\sigma_{s}$ is the system's uncertainty to resolve the phase. Here, $\sigma_{s}$ cannot be derived analytically. Zedel et al. (1996), working on a single beam pulse-to-pulse coherent Doppler profiler that is based on the same principle as the ADV examined here, found $\sigma_{s}$ to be 0.067 , corresponding to a phase uncertainty $\pm 6^{\circ}$. However, their system used different transducers $(1.7 \mathrm{MHz})$ and was equipped with a 12-bit $\mathrm{A} / \mathrm{D}$ converter, ensuring better resolution of the phase signal; therefore, application of Eq. (10) requires a new $\sigma_{s}$ value suitable to the resolution of the ADV (discussed in section $4 \mathrm{a}$ ).

Coherent Doppler acoustic systems are sensitive to Doppler phase noise, which causes a broadening of the Doppler spectral peak. This results in an error in the radial velocity estimated to be (Brumley et al. 1991) 


$$
\sigma_{D}^{2}=\frac{\pi^{-1 / 2}}{16} \frac{c^{2} B}{f^{2} M \tau},
$$

where $B$ (total Doppler bandwidth broadening) is the rms (i.e., $B^{2}=B_{r}^{2}+B_{t}^{2}+B_{d}^{2}$ ) of the three individual contributions (Cabrera et al. 1987). These are broadening due to finite residence time $B_{r}$, turbulence within the sample volume $B_{t}$, and beam divergence $B_{d}$. Finally, $M$ is the number of acoustic pulses averaged for the estimation of the radial velocity.

Noise due to residence time is attributed to the fact that during successive acoustic pingings, some scatterers will have moved out of the sample volume, while new particles will have been introduced. The residence time of the scatterers is of the order $d / U$. The Doppler spectral broadening due to this effect is estimated to be (Cabrera et al. 1987)

$$
B_{r}=0.2 \frac{U}{d}
$$

where $d$ is the transverse size of the ADV's sample volume $\left(\simeq d_{u}\right)$ and $U$ is the mean horizontal speed.

Turbulence at spatial scales of the order of the sample volume $d$ or smaller causes the scatterers to have a distribution of velocities. The spectral broadening due to this effect is estimated to be (Cabrera et al. 1987)

$$
B_{t}=2.4 \frac{f(\epsilon d)^{1 / 3}}{c},
$$

where $\epsilon$ is the turbulence dissipation rate.

Another source of spectral broadening is due to small variation across the beam of the normal to the transducer (beam divergence). This has been estimated to be (Cabrera et al. 1987)

$$
B_{d}=0.84 \sin (\Delta \theta) \frac{f U_{c}}{c},
$$

where $\Delta \theta$ is the bistatic angle $\left(15^{\circ}\right)$. The coefficients in Eqs. (12) to (14) are based on assumptions regarding the geometry of the sensing volume (B. H. Brumley et al. 1987, unpublished manuscript, cited in Cabrera et al. 1987; E. A. Terray 1996, personal communication).

Finally, the variance contribution due to the shear of the mean flow within the sample volume $d$ is estimated to be (Lhermitte and Lemmin 1994)

$$
\sigma_{u}^{2}=\frac{(\Delta U)^{2}}{3},
$$

where $\Delta U$ is the variation of the velocity in the sample volume [i.e., $U(z)-U(z+d)$ ]. Assuming a turbulent boundary layer flow, where the law of the wall (i.e., logarithmic distribution) is valid, then

$$
\Delta U=\frac{u_{*}}{\kappa} \ln \left(1+\frac{d_{w}}{z}\right) .
$$

Equation (16) indicates that the error due to shear of the mean flow varies with height above the boundary, and it is expected to be significant only at positions close to the boundary layer and particularly at heights similar to that of the sample volume.

The error due to the ability of the sensor to resolve phase differences [Eq. (10)] is independent of flow conditions. It depends only on pulse length, which is set by the velocity range (see Table 1). The noise due to Doppler broadening [Eq. (11)] and mean velocity gradient [Eq. (15)] is flow related. The relative importance of the terms comprising these two components was examined using Eqs. (11)-(16), assuming open channel flow with a logarithmic distribution of the mean flow and a vertically varying turbulence dissipation given by $\epsilon=u_{*}^{3}(1-z / h) /(\kappa z)$, where $\kappa$ is the von Kármán's constant (0.4). Calculations were carried out for three flow conditions with bottom shear velocities $0.50,0.75$, and $1 \mathrm{~cm} \mathrm{~s}^{-1}$, respectively. The results are shown in Fig. 3 as the ratio of each noise source to the total error combined from the flow-related sources. Evidently, the most significant noise term is that due to turbulence, while the noise due to mean velocity shear becomes equally important at elevations close to the boundary $(z$ $<0.1 \mathrm{~h}$ ). Higher in the water column the turbulence reduces, as does the mean velocity shear, so that noise terms associated with mean velocity magnitude dominate. However, in absolute values these noise terms are not significant and can be ignored, at least in boundary layer applications.

\section{Experimental equipment and measurement techniques}

\section{a. Open-channel flume}

The experiments were carried out in a tilting flume $17 \mathrm{~m}$ long, $60 \mathrm{~cm}$ wide, and $30 \mathrm{~cm}$ deep (Butman and Chapman 1989), which is located in the Coastal Research Laboratory at the Woods Hole Oceanographic Institution. The water is circulated by a centrifugal pump, and its temperature is controlled within $\pm 0.5^{\circ} \mathrm{C}$, ensuring accuracy in speed of sound and, consequently, in acoustically measured flow speed, within $\pm 0.03 \%$ (Clay and Medwin 1977). The pump runs at a constant speed and pumps up to $0.095 \mathrm{~m}^{3} \mathrm{~s}^{-1}$ at $13.7 \mathrm{~m}$ total dynamic head. Mean flow speed and water depth in the runway are controlled by the discharge rate and a vertical weir system at the terminus of the flume raceway. The channel bed is covered by PVC sheets simulating a hydraulically smooth bed, while a settling chamber and screens at the entrance of the channel prevents the occurrence of large-scale flow disturbances. The flume has been extensively tested (Trowbridge et al. 1989) and can produce approximately one-dimensional flow for water depths less than roughly $15 \mathrm{~cm}$. Averaging times of approximately $6 \mathrm{~min}$ are sufficient to produce estimates of mean velocities that are within a few percent of the true mean (Trowbridge et al. 1989). 


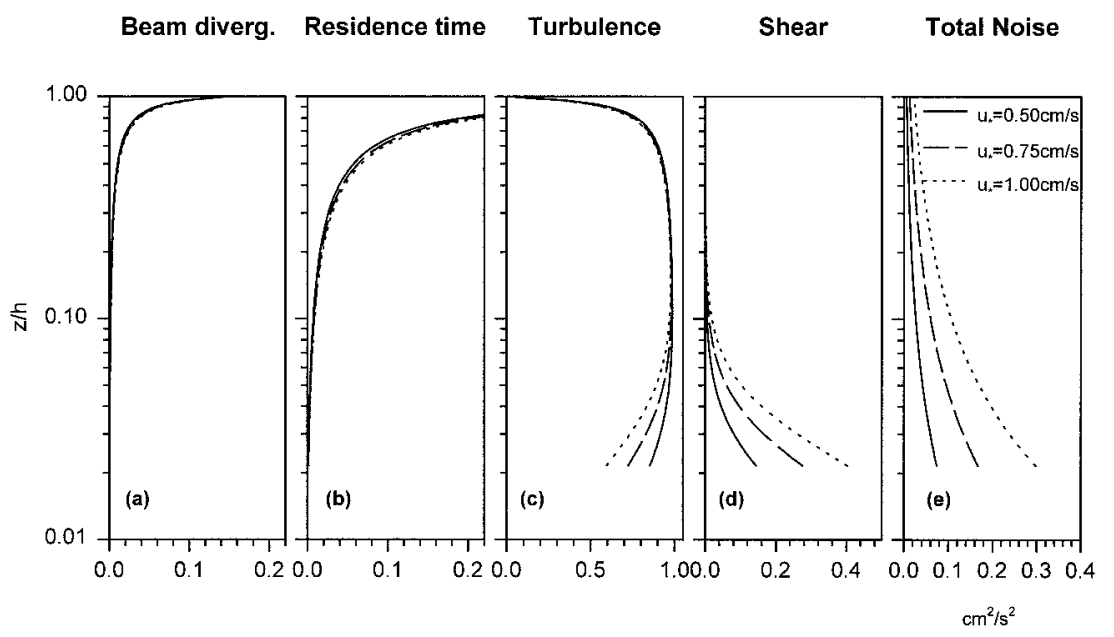

FIG. 3. Vertical variation of the contribution of the various flow-related noise sources, (a)(d), and the total noise variance (e) associated with measurements of current velocity using acoustic Doppler techniques. The case of three different flow conditions (shear velocities) is shown. Calculations are based on Eqs. (11)-(16) for a sample volume size $d=0.6 \mathrm{~cm}$.

The mean velocity $U$ distribution in a steady, onedimensional, open-channel flow above a smooth bottom is given by the general semiempirical expression (Nezu and Rodi 1986)

$$
U(z)=u_{*} f\left(z_{+}\right)+u_{*} \mathcal{F}(\xi),
$$

where $u_{*}$ is the shear velocity, $z_{+}$is $z u_{*} / \nu, \xi$ is $z / h$, and $f\left(z_{+}\right)$and $\mathcal{F}(\xi)$ are the wall and wake correction functions, respectively. Well outside the viscous sublayer $\left(z_{+}\right.$ $>50$ ) and neglecting the wake correction, the following commonly used logarithmic approximation to the mean velocity profile above a smooth wall may be obtained:

$$
U(z)=\frac{u_{*}}{\kappa} \ln \left(\frac{z u_{*}}{\nu}\right)+A,
$$

where $A=5.5$.

Root-mean-square turbulent velocity fluctuations in a one-dimensional open-channel flow above a smooth bottom, as in the experimental facility used in this study, are expected to follow the expressions suggested by Nezu and Rodi (1986):

$$
\left\langle u^{\prime 2}\right\rangle^{1 / 2}=u_{*} D_{u} \exp \left(-\lambda_{u} \frac{z}{h}\right)
$$

and

$$
\left\langle w^{\prime 2}\right\rangle^{1 / 2}=u_{*} D_{w} \exp \left(-\lambda_{w} \frac{z}{h}\right),
$$

where $D_{u}, \lambda_{u}, D_{w}, \lambda_{w}$ are empirical constants with values $2.26,0.88,1.23$, and 0.67 , respectively. Equations (19) and (20) are valid for the region in which $z u_{*} / \nu$ is greater than about 50 and $z / h$ is less than 0.6 .

The vertical variation of the Reynolds stress can be extracted by balancing the mean forces and momentum fluxes. For elevations greater than $50 \nu / u_{*}$ it can be shown that

$$
-\left\langle u^{\prime} w^{\prime}\right\rangle \simeq u_{*}^{2}\left(1-\frac{z}{h}\right)
$$

The above equations were found satisfactory for describing the flow conditions in the flume used for this study (see Trowbridge et al. 1989).

\section{b. Laser Doppler velocimeter}

The LDV system used in this study was a forward scattering device similar to the field laser device described by Agrawal and Belting (1988). It measures flow velocities along two axes using an $8-\mathrm{mW}$ helium-neon laser beam split into three beams that intersect at the measurement volume. The sample volume is approximately ellipsoidal, with a long axis in the transverse direction of $1 \mathrm{~mm}$ in length and a circular cross section of $0.3 \mathrm{~mm}$ in diameter. This device measures two velocity axes $(X$ and $Y)$ at $\pm 42.5^{\circ}$ to the horizontal in a plane normal to the cross-sectional line of the flume. Similar to the ADV, the LDV-measured velocities along the $X$ and $Y$ beams $\left(u_{x}\right.$ and $u_{y}$, respectively) consist of a true flow and a noise term. Geometrical corrections in post processing are used to rotate to local bed-parallel $u$ and bed-normal $w$ components:

$$
u=\frac{\left(\tilde{u}_{x}+U_{\mathrm{nx}}\right)+\left(\tilde{u}_{y}+U_{\mathrm{ny}}\right)}{2 \cos (42.5)}
$$

and

$$
w=\frac{\left(\tilde{u}_{x}+U_{\mathrm{nx}}\right)-\left(\tilde{u}_{y}+U_{\mathrm{ny}}\right)}{2 \sin (42.5)},
$$

where the tilde denotes real flow velocities and $U_{\mathrm{nx}}$ and 
$U_{\text {ny }}$ are the noise components for the $X$ and $Y$ directions, respectively. The resolution of the LDV as defined by the sampling scheme is $0.25 \mathrm{~cm} \mathrm{~s}^{-1}$ (Butman and Chapman 1989).

If $U_{\mathrm{nx}}$ and $U_{\mathrm{ny}}$ are uncorrelated, then the turbulence statistics calculated using the measured velocities can be expressed as functions of the real value of the parameter and an error associated with the noise component:

$$
\begin{aligned}
\left\langle u^{\prime} w^{\prime}\right\rangle & =\left\langle\tilde{u}^{\prime} \tilde{w}^{\prime}\right\rangle+\frac{\left\langle U_{\mathrm{nx}}^{2}\right\rangle-\left\langle U_{\mathrm{ny}}^{2}\right\rangle}{4 \cos (42.5) \sin (42.5)}, \\
\left\langle u^{\prime 2}\right\rangle & =\left\langle\tilde{u}^{\prime 2}\right\rangle+\frac{\left\langle U_{\mathrm{nx}}^{2}\right\rangle+\left\langle U_{\mathrm{ny}}^{2}\right\rangle}{4 \cos ^{2}(42.5)},
\end{aligned}
$$

and

$$
\left\langle w^{\prime 2}\right\rangle=\left\langle\tilde{w}^{\prime 2}\right\rangle+\frac{\left\langle U_{\mathrm{nx}}^{2}\right\rangle+\left\langle U_{\mathrm{ny}}^{2}\right\rangle}{4 \sin ^{2}(42.5)} .
$$

The above equations demonstrate that the error in the Reynolds stress as measured by the LDV depends on how well the noise is balanced between the two measuring axes [Eq. (24)]. The error in variance is similar for both the horizontal and vertical velocities. A detailed description of the nature of the errors involved in measuring flow characteristics with the laser Doppler technique is beyond the scope of this paper. More details regarding the errors involved in measuring flow with laser Doppler systems can be found in George and Lumley (1973) and Buchhave et al. (1979).

\section{c. "Ground truthing" of flow characteristics}

Since the objective of the present study is to evaluate the performance of the ADV for measuring flow and turbulence characteristics, there is a need for comparing the measurements to a ground truth value. In earlier evaluation studies (Kraus et al. 1994; Lohrmann et al. 1994; Lohrmann et al. 1995), measurements obtained using an ADV were compared directly to either LDV measurements (Fig. 1a) or data collected with vectoraveraging current meters (Anderson and Lohrmann 1995). However, there is no error-free velocity measurement device. Therefore, measurements of mean water velocity, variance, or covariance with in situ sensors cannot be considered as a reference known to a satisfactory degree of accuracy. A ground truth needs to be invoked as a means to determine that one method gives more accurate results than another. As an example, simple intercomparison of the Reynolds stress values derived from the ADV and LDV sensors, respectively, although in general agreement with the data presented by Lohrman et al. (1994) (see Fig. 1a), suggested that the ADV underestimates Reynolds stresses by a factor of 2 compared with the LDV. However, the validity of such conclusion depends upon the degree of accuracy of the measurements obtained by the LDV.
This problem can be overcome by using two independent measuring methods with known relationships between the noise and "true" flow terms. Considering the ADV and LDV as the two independent measuring methods, flow measurements obtained using these sensors can be utilized to estimate the true flow statistics. This approach requires that both sensors sample the same volume within the fluid or that flow statistics are identical at both sampling locations. In such a case, the true flow signal (denoted by tilde) is a common parameter in the pairs of Eqs. (5) and (24), (6) and (25), and (8) and (26), respectively. The right term in all of those equations is calculated using the data recorded by the ADV and LDV sensors, respectively, while the linear system of these six equations can be solved to derive $1)$ the true flow statistical parameters $\left(\left\langle\tilde{u}^{\prime 2}\right\rangle,\left\langle\tilde{w}^{\prime 2}\right\rangle\right.$ and $\left.\left\langle\tilde{u}^{\prime} \tilde{w}^{\prime}\right\rangle\right)$ and 2) the noise levels for the $\operatorname{ADV}\left(\sigma_{t}^{2}\right)$ and the $\operatorname{LDV}\left(\left\langle U_{\mathrm{nx}}^{2}\right\rangle,\left\langle U_{\mathrm{ny}}^{2}\right\rangle\right)$.

It should be noted that application of the ground truthing is based on the assumption that the noise variance along the three acoustic beams is equal in magnitude and uncorrelated (see Figs. 1b and 1c, respectively). Violation of this assumption will result in a linear system of six equations but with eight unknowns since each acoustic beam will have a different noise variance. In such a case, the above technique is not valid.

\section{d. Experimental setup}

Both the transmitter and receiver units of the laser system were cantilevered, at either side of the flume, from a horizontal aluminum beam. The center of the beam was attached to a vertical stand fixed to a carriage trolley allowing the system to be moved along the flume channel. The horizontal beam was extended some 70 $\mathrm{cm}$ from either side of its support center. The ADV was attached to the beam at the central point (Fig. 2b). Although the two sensors were arranged so they measured flow velocities at the same elevation above the flume bed, the LDV sample volume was positioned at approximately $5 \mathrm{~cm}$ downstream from the ADV measuring volume (Fig. 2a). Despite this small horizontal displacement, the statistical properties of the turbulence are expected to be the same at both locations. Data were collected for $6 \mathrm{~min}$ at a sampling frequency of $25 \mathrm{~Hz}$ for both sensors. Logging was initiated manually for both instruments; a synchronization error less than approximately $0.25 \mathrm{~s}$ was believed to occur at the start of the data. Three experiments (denoted A, B, and C) were undertaken to determine the ability of the sensor to measure flow characteristics, while another experiment in still water was undertaken to estimate noise levels due to the ADV electronics $\left(\sigma_{m}^{2}\right)$.

Experiment A focused on investigating the performance of the ADV for a variety of flow conditions. Therefore, flow depth $h$ and height of measurement $z$ were altered for each run during the experiment. In addition, to evaluate the effect of the velocity range (Table 
TABLE 2. Setup during experiment $\mathrm{A} ; h$ is the water depth, $z$ is the current velocity measurement height above the bottom, $U$ is the mean horizontal velocity as obtained from the ADV, and range refers to the setting of the ADV sensor during the experiment. The correlation coefficient (COR) between the transmitted and received pulses shown is averaged over the whole run $(6 \mathrm{~min})$ and all three beams.

\begin{tabular}{cccccc}
\hline \hline $\begin{array}{c}\text { Run } \\
\text { number }\end{array}$ & $h(\mathrm{~cm})$ & $z(\mathrm{~cm})$ & $U\left(\mathrm{~cm} \mathrm{~s}^{-1}\right)$ & $\begin{array}{c}\text { Range } \\
\left(\mathrm{cm} \mathrm{s}^{-1}\right)\end{array}$ & COR \\
\hline 1 & 18.0 & 4.07 & 7.93 & 30 & 97 \\
2 & 18.0 & 4.05 & 7.91 & 10 & 96 \\
3 & 25.0 & 4.15 & 28.98 & 30 & 94 \\
4 & 25.0 & 4.13 & 29.09 & 100 & 97 \\
5 & 25.0 & 4.13 & 29.12 & 250 & 97 \\
6 & 25.0 & 8.12 & 32.09 & 30 & 92 \\
7 & 16.5 & 2.21 & 28.80 & 30 & 92 \\
8 & 16.5 & 2.16 & 28.84 & 100 & 96 \\
9 & 16.5 & 2.18 & 27.36 & 250 & 52 \\
10 & 19.4 & 3.23 & 24.30 & 30 & 92 \\
11 & 19.4 & 3.20 & 24.50 & 100 & 93 \\
12 & 19.4 & 3.20 & 24.41 & 250 & 97 \\
13 & 19.4 & 3.20 & 24.75 & 10 & 86 \\
14 & 19.4 & 3.23 & 24.84 & 3 & 76 \\
15 & 25.0 & 3.09 & 18.06 & 3 & 82 \\
16 & 25.0 & 3.10 & 17.63 & 10 & 90 \\
17 & 25.0 & 3.09 & 17.78 & 30 & 92 \\
18 & 25.0 & 3.10 & 17.53 & 100 & 91 \\
19 & 18.2 & 3.09 & 16.52 & 30 & 92 \\
20 & 18.2 & 3.09 & 16.48 & 100 & 91 \\
21 & 18.2 & 3.09 & 16.77 & 10 & 91 \\
22 & 25.5 & 3.09 & 7.46 & 10 & 95 \\
23 & 25.5 & 3.10 & 7.40 & 30 & 93 \\
24 & 20.0 & 1.09 & 6.30 & 10 & 95 \\
\hline & & & & &
\end{tabular}

1), data were collected using different velocity range settings. The flow conditions and velocity ranges used during this experiment are listed in Table 2. In experiment B, both ADV and LDV sensors were used to obtain a vertical profile of the flow characteristics. Mean flow depth was $16.2 \mathrm{~cm}$ and some 14 measurements were undertaken at elevations above the flume bed that varied between 0.76 and $4.7 \mathrm{~cm}$.

In experiment $\mathrm{C}$, only the $\mathrm{ADV}$ sensor was used due to failure of the LDV. The experiment involved measurements of variation of flow parameters with elevation above the flume bed in a similar way as for experiment B except that no LDV data were available, and thus no ground truth flow values could be obtained. Three profiles were obtained $\left(\mathrm{C}_{1}, \mathrm{C}_{2}\right.$, and $\left.\mathrm{C}_{3}\right)$ with different flow conditions (Table 3 ).

Prior to data analysis and calculation of flow statistics, the data were corrected for sensor misalignment. The misalignment correction procedure involved rotation along the horizontal axis (perpendicular to the $u w$ plane) so the mean vertical velocity was equal to zero. The averaged rotation angles for all runs, were $0.6^{\circ}$ and $0.4^{\circ}$ for the LDV and ADV sensors, respectively. The rotation procedure is based on the assumption that there is no real mean vertical flow (i.e., secondary flow). Although this might not be the case during the experimental work, the error introduced by such a rotation is insignificant. In Reynolds stress estimations, for ex-
TABLE 3. Mean water depth $h$ and range and number of elevations above the flume bed where data were collected during experiment $\mathrm{C}$.

\begin{tabular}{ccccc}
\hline \hline Test & $h(\mathrm{~cm})$ & $z_{\min }(\mathrm{cm})$ & $z_{\max }(\mathrm{cm})$ & $\begin{array}{c}\text { Number of } \\
\text { elevations }\end{array}$ \\
\hline $\mathrm{C}_{1}$ & 25.0 & 0.6 & 11.2 & 11 \\
$\mathrm{C}_{2}$ & 19.5 & 0.5 & 6.9 & 9 \\
$\mathrm{C}_{3}$ & 15.0 & 0.3 & 3.3 & 9 \\
\hline
\end{tabular}

ample, the error is a function of the misalignment angle and the difference between the variances of the horizontal and vertical velocity components. Using the collected experimental data it was calculated that the maximum error in Reynolds stress estimates would be $1.3 \%$ and $0.9 \%$ of the vertical velocity variance, which translates to 0.04 and $0.01 \mathrm{~cm}^{2} \mathrm{~s}^{-2}$ for the LDV and ADV sensors, respectively.

\section{Results/discussion}

\section{a. Still water experiment}

The sampling error $\sigma_{m}^{2}$ due to the sensor's ability to resolve the Doppler phase shift and electronically induced noise was determined experimentally by obtaining measurements in still water. One set of data was collected for each instrument velocity range setting (Table 1). The variance of the recorded signal is shown in Table 1 . This approach is not considered very accurate because at no-flow conditions the density of scatterers in the water will be minimal. However, the correlation coefficient between transmitted and received pulses was approximately 0.75 , which is greater than the minimum value (0.70) required for accurate measurements (SonTek 1995). Table 1 shows that this noise term (sampling error) tends to increase with velocity range setting. The equivalent uncertainties in phase detection $\sigma_{s}^{2}$ were calculated using Eq. (10), and the values derived are also listed in Table 1. Surprisingly, even the phase uncertainties appear to be a function of the velocity range setting of the instrument. The unusually high noise level observed during experiment $A$ (see section $4 b$ ), for the nominal velocity range of $100 \mathrm{~cm} \mathrm{~s}^{-1}$, is also present in these data.

\section{b. Experiment $A$}

During this experiment, 24 runs were carried out in which instantaneous flow velocities were measured at different elevations above the flume bed for various flow conditions and ADV velocity range settings. Calculated mean flow was based on averaging the 6-min-long instantaneous values. These mean flows are considered free of any of the noise described above since averaging cancels the effects of the noise. Therefore, mean downstream $\langle u\rangle$ flows measured by ADV and LDV can be intercompared directly. This is shown in Fig. 4a where the two sensors appear to agree with all data points 
Mean Velocities

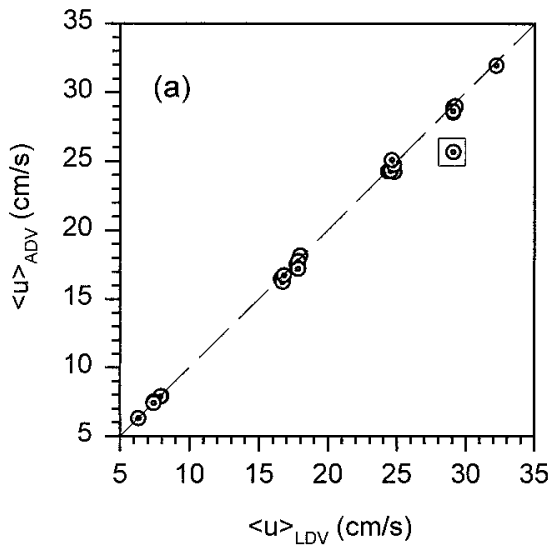

Horizontal Variance

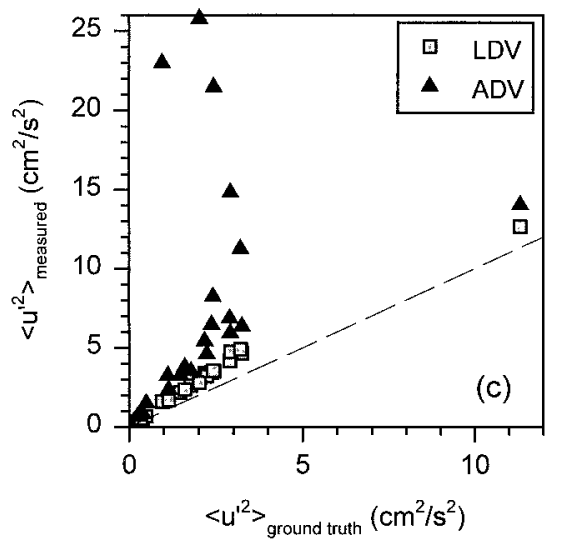

Reynolds Stress

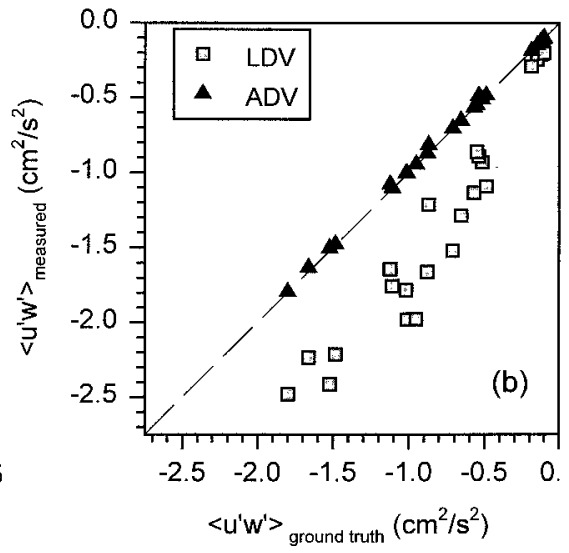

Vertical Variance

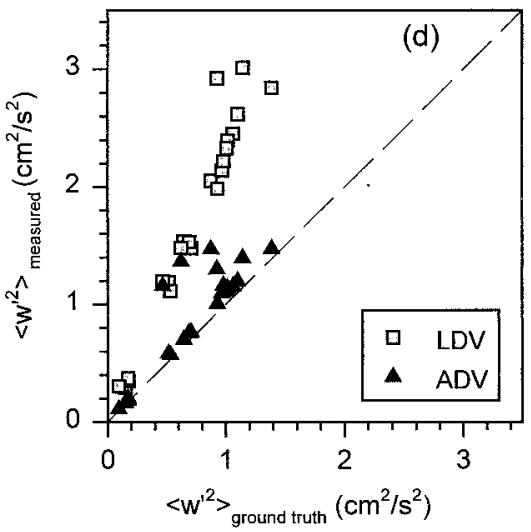

FIG. 4. Statistical parameters of flow calculated from ADV and LDV measurements during experiment A. (a) Intercomparison of mean horizontal velocity measured by the two sensors. (b) Reynolds stress values measured with the ADV (triangles) and the LDV (squares) plotted against the real Reynolds stress value (ground truth) of the flow. Variance of horizontal (c) and vertical velocities (d) measured with the ADV and LDV (triangles and squares, respectively) plotted against the real variances of the flow (ground truth). (Note: The straight line is the 1:1 curve.)

(except for run 9) lying along the 1:1 line. The mean value obtained by the ADV during run 9 (velocity range setting $250 \mathrm{~cm} \mathrm{~s}^{-1}$, shown enclosed in a square in Fig. 4a) presents an anomaly to the above agreement. The correlation coefficient between the transmitted and received pulses for run 9 was 0.52 (see Table 2), which is below the 0.70 value required for reliable flow measurements (SonTek 1995). A possible explanation of the anomalous behavior during run 9 is the failure of the sensor to adjust its staggered transmit delay times to resolve speed ambiguities at the ranges used during the experiments (R. Cabrera 1996, personal communication). Run 9 has been excluded from any analysis presented hereafter. Regression analysis showed that the ADV underestimates the mean flow by $1 \%$ relative to the LDV. The mean square error between the two sensors was $0.56 \mathrm{~cm} \mathrm{~s}^{-1}$.
Although part of the underestimation of the mean flow speed by the ADV could be explained by the size of the sample volume (some 30 times larger than that of the LDV, in the vertical direction), a more likely explanation is that the LDV and ADV sample volumes were at slightly different heights. Within the sample volume, the mean velocity is logarithmically distributed; thus simple arithmetic averaging will give a biased estimate of the volume integrated velocity. Errors of this type depend on the degree of shear. For the present experimental runs, this was calculated to be of the order of $-0.02 \%$ to $-0.1 \%$, which is significantly lower than the observed $1 \%$. The majority of the observed error is attributed to problems in aligning the sample volume of the sensors at the same level. Using a logarithmic velocity distribution and the shear stress values calculated for each particular run (see below), the size of the ver- 
tical misalignment required to produce an error of $1 \%$ was calculated. It was found consistent with the ADV being located higher than the LDV by an average 1.8 mm (approximately $1 / 5$ of $d_{w}$ ) with a $95 \%$ confidence interval of $0.25 \mathrm{~mm}$. Such an error is likely considering the difficulties in vertically aligning the two sensors, especially since the ADV sample volume is not visible, unlike the LDV sample volume.

"Real" turbulence statistics of the flow have been estimated using the ground truth method described in section 3c. The calculated values of covariance (Reynolds stress) and variances are compared to the corresponding ground truth values in Figs. $4 \mathrm{~b}, 4 \mathrm{c}$, and $4 \mathrm{~d}$, respectively.

Reynolds stress values calculated from the ADV time series and the values estimated with the ground truthing procedure are in good agreement (Fig. 4b). In contrast, the LDV-derived Reynolds stress values are approximately twice as larger as the ground truth values. Equation (24) reveals that Reynolds stress estimation using the LDV will be free of any error if the noise levels in both beams are equal in magnitude and uncorrelated with each other. Spectral analysis of the LDV velocities measured along the laser beams revealed that the noise level on the $Y$ beam was approximately twice that of the $X$ beam. This was the reason for the anomalously large Reynolds stress values calculated using the LDV records.

The Reynolds stress $-\left\langle u^{\prime} w^{\prime}\right\rangle$ derived using the ADV is on average $1.7 \%( \pm 0.96 \%$ at $95 \%$ confidence interval) greater than the ground truth values (Fig. $4 b$ ). This error includes the values from the three runs with a velocity range of $100 \mathrm{~cm} \mathrm{~s}^{-1}$, which exhibited a higher noise level. It is characteristic that all other runs exhibit an average error of $0.9 \%$ (with $95 \%$ confidence interval of $\pm 0.15 \%$ ). This error is consistent with the uncertainty in vertical alignment of the ADV and LDV sample volumes.

The LDV overestimated both horizontal and vertical velocity variances by approximately $50 \%$ and $120 \%$, respectively. The ADV, on the other hand, was better in measuring vertical velocity variances (Fig. 4d) than in measuring horizontal velocity variances (Fig. 4c). These findings agree with the fact that any noise level along the ADV measuring beam is enhanced by approximately 11 times along the horizontal and only 0.35 times along the vertical due to the sensor geometry [Eqs. (5)-(8)]. Additionally, the agreement of the ADV-measured variances with those derived using the ground truthing method is not always the same. There are some points that lie outside the suggested trend. These points are from data runs with increased noise levels (i.e., high velocity range settings; see section $4 \mathrm{f}$ ). This behavior is characteristic only of the variances (Figs. $4 \mathrm{c}$ and $4 \mathrm{~d}$ ) and not of the covariances (Fig. 4b), thereby confirming the validity of the assumptions used for deriving Eqs. (5)-(8), which pertain to the effect of noise to the moments of the measured flow.

\section{Velocity Profiles}

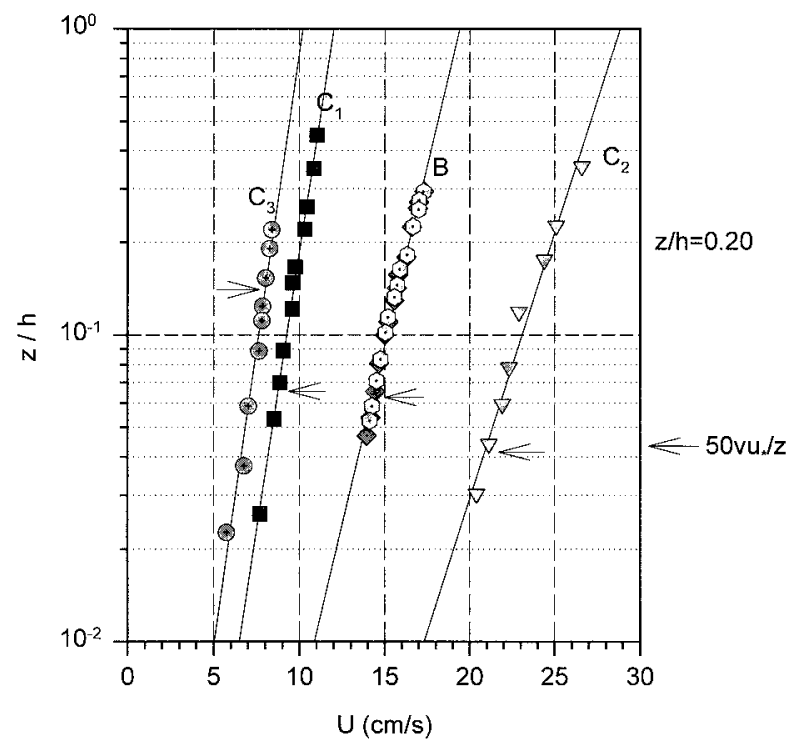

FIG. 5. Mean velocity profiles measured during experiments B and C. Straight lines represent regression analysis through all the points of each profile.

\section{c. Experiment B}

The vertical distribution of the mean velocity obtained by both ADV and LDV sensors is shown with a line fitted through the whole of the data points, in Fig. 5. Characteristically, the velocity profile exhibits a deviation from the theoretical distribution described by open-channel flow theory (i.e., Nezu and Rodi 1986) for $z / h<0.1$. There the profile exhibits a change in slope. Similar changes were observed in velocity distribution measurements in a flume by Lhermitte and Lemmin (1994), which were attributed to the averaging effect on the sharp mean velocity gradients near the boundary. In the present experiment, however, this deviation is observed even for the mean velocities measured using the LDV, which has a much smaller sample volume. This suggests that the deviation is a real phenomenon due to either certain flow conditions in open channels or the presence of secondary roughness elements in the flume. The agreement of the logarithmic profile of the mean velocity obtained with the ADV and LDV, respectively, suggests that the ADV can be used for measuring mean currents even at elevations close to the boundary $(0.76 \mathrm{~cm})$.

The logarithmic distribution of mean velocity, Eq. (18), was fitted to 1) velocity data points obtained at elevations between $0.1 h$ (i.e., $2 \mathrm{~cm}$ ) and $0.2 h$ (i.e., above the region where the change in the slope of the profile occurs) and 2) data collected at elevations between $50 \nu / u_{*}$ and $0.2 \mathrm{~h}$. The derived bottom shear velocity values were 0.84 and $0.74 \mathrm{~cm} \mathrm{~s}^{-1}$ for the two fits, respectively. These values have been used to predict 


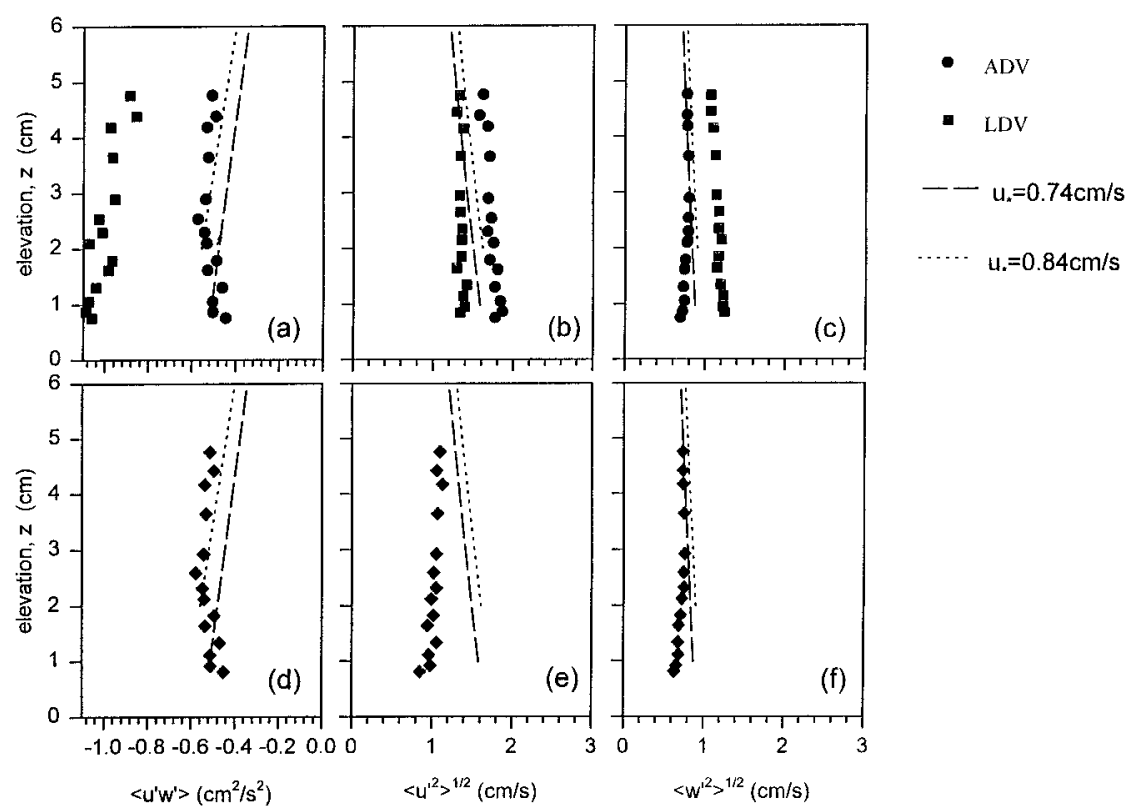

FIG. 6. Experiment B. Vertical variation of Reynolds stress and turbulence intensities. Circles: measurements with ADV. Squares: measurements with the LDV. Lines: Eqs. (19), (20), and (21). Diagrams (a), (b), and (c) present raw collected data, whereas the ground truth values are shown in diagrams (d), (e), (f).

the turbulence intensities and Reynolds stress vertical distribution according to Eqs. (19), (20), and (21), as shown in Fig. 6. In the figure, predictions based on $u_{*}$ $=0.74 \mathrm{~cm} \mathrm{~s}^{-1}$ are shown as dashed lines, while dotted lines represent predictions based on $u_{*}=0.84 \mathrm{~cm} \mathrm{~s}^{-1}$. The top row of diagrams (a, b, and c) represents the vertical variation of the turbulence intensities and covariance as these have been measured by the ADV (circles) and LDV (squares), respectively. The bottom row $(\mathrm{d}$, e, and $\mathrm{f})$ shows the vertical variation of the same parameters as these have been derived by the ground truthing procedure.

Open-channel flow measurements (Nezu and Rodi 1984) predict a decrease of the Reynolds stress with elevation above the flume bed [Eq. (21)]. This is true for the data obtained by both ADV and LDV (Fig. 6a) for elevations greater than $2 \mathrm{~cm}(\sim 0.1 h)$. This range of data agrees with the theoretical curve obtained using the shear velocity determined by fitting the log profile to the upper section of the profile only (i.e., $u_{*}=0.84$ $\mathrm{cm} \mathrm{s}^{-1}$ ). For $z<2 \mathrm{~cm}$, the Reynolds stress measured by the ADV exhibits a decrease in value; the LDVderived Reynolds stress values, although greater in magnitude due to noise contribution, exhibit a sudden decrease but then start increasing again toward the bed.

These anomalous values of Reynolds stress occur at measurements obtained at elevations less than $2 \mathrm{~cm}$. This elevation coincides with the elevation where the mean velocity profile changes (increases) in slope (Fig. 5). Such a behavior could be explained if it is assumed that the flow responds to two roughness elements (e.g., Smith and McLean 1977). In such a case the smaller element determines the vertical distribution of $U$ near the bottom, while the larger roughness element defines the profile slope at elevations greater than $2 \mathrm{~cm}$. The LDV-derived Reynolds stress distribution, although incorrect in terms of magnitude, exhibits a break at $z=$ $2 \mathrm{~cm}$, and thereafter the data points show an increase with decreasing elevations above the bottom, as expected according to Eq. (21). Such a $\left\langle u^{\prime} w^{\prime}\right\rangle$ distribution also supports the case of the presence of two roughness elements. In contrast to the LDV, the ADV-derived Reynolds stresses are monotonically decreasing for $z<$ $2 \mathrm{~cm}$. This is believed to be related to the effect of the size of the sample volume $d$. It was shown in section 2a that eddies smaller than $2.2 \mathrm{~cm}$ in the vertical and $1.5 \mathrm{~cm}$ in the horizontal are not fully resolved by the ADV sensor. These lengths are similar to the height where the anomalous behavior in ADV-derived $\left\langle u^{\prime} w^{\prime}\right\rangle$ values is observed, and therefore it could be attributed to the effect of the size of the sample volume. However, this deviation of the measured values is comparable to that seen in the plots of the experimental data collected by Nezu and Rodi (1984) and Trowbridge et al. (1989) using LDV sensors with significantly smaller sample volume.

The measured (ADV) turbulence intensity of the horizontal component is higher than the values predicted by Eq. (10). However, the increase in intensity with decreasing elevation above the flume bed [as predicted by Eq. (19)] is exhibited quite clearly by the collected data (Fig. 6b). This increased magnitude is due to increased noise levels in the horizontal component due to the geometry of the sensor. However, correcting these 
TABLE 4. Estimates of bottom shear velocity using the log-profile [Eq. (18)] and Reynolds stress [Eq. (21)] techniques, respectively. The errors in the estimate are shown with a confidence limit of $95 \%$.

\begin{tabular}{cccrccr}
\hline \hline & \multicolumn{2}{c}{ Log-profile technique } & & \multicolumn{2}{c}{$\begin{array}{c}\text { Reynolds stress } \\
\text { technique }\end{array}$} \\
\cline { 2 - 3 } \cline { 6 - 6 } Exp. & $u_{*}\left(\mathrm{~cm} \mathrm{~s}^{-1}\right)$ & $r^{2}$ & $N$ & & $u_{*}\left(\mathrm{~cm} \mathrm{~s}^{-1}\right)$ & $N$ \\
\hline $\mathrm{B}$ & $0.738 \pm 0.562$ & 0.978 & 16 & & $0.782 \pm 0.014$ & 12 \\
$\mathrm{C}_{1}$ & $0.446 \pm 0.120$ & 0.955 & 5 & & $0.435 \pm 0.077$ & 9 \\
$\mathrm{C}_{2}$ & $0.912 \pm 0.108$ & 0.960 & 5 & & $1.019 \pm 0.061$ & 7 \\
$\mathrm{C}_{3}$ & 0.385 & - & 2 & & $0.356 \pm 0.096$ & 3 \\
\hline
\end{tabular}

values (Fig. 6e) makes the horizontal intensity smaller than the predicted one, whereas the value in the vertical remains almost constant. On the contrary, the vertical component (Fig. 6f) seems to agree well with the theory. The agreement is best at heights greater than $3 \mathrm{~cm}$. This is assumed to be for the same reasons as explained above for the Reynolds stress.

\section{d. Experiment $C$}

During these experiments, the vertical distribution of the mean flow was defined by ADV measurements for the three different flow conditions shown in Table 3. The velocity profiles are shown in Fig. 5. It is characteristic that the change in the slope of the profile observed during experiment $\mathrm{B}$ is present also in experiments $C_{1}$ and $C_{2}$. Bottom shear velocities for the experiments were calculated using 1) the log-profile technique assuming smooth wall conditions [Eq. (18)] and 2) the measured $\left\langle u^{\prime} w^{\prime}\right\rangle$ values using Eq. (21) (Reynolds stress technique). The bottom shear velocity value obtained using the former technique is the result of a least square fit of Eq. (18) to the experimental data, while the value obtained with the Reynolds stress method is an averaged value of $u_{*}$ obtained from $\left\langle u^{\prime} w^{\prime}\right\rangle$ measurements at $z>50 \nu / u_{*}$. The results of the analysis are shown in Table 4 with the results of the same analysis for experiment $\mathrm{B}$. The maximum difference $(10 \%)$ was found to exist for experiment $\mathrm{C}_{1}$. However, this difference is within the confidence interval of the estimate based on the log-profile technique. This agreement shows the ability of the ADV to accurately measure Reynolds stress.

\section{e. Spectral characteristics}

The results presented in the above section are based on the analysis of time series data. The spectral characteristics of the vertical and horizontal flow as measured by the ADV sensor are examined here. In particular, the spectra from two typical runs with significantly different Reynolds numbers are presented in Fig. 7.

Figure 7a displays the horizontal (dotted line) and vertical (solid line) components of the velocity wavenumber spectra calculated by both the ADV and the LDV measurements at $z=3.1 \mathrm{~cm}$. The flow conditions
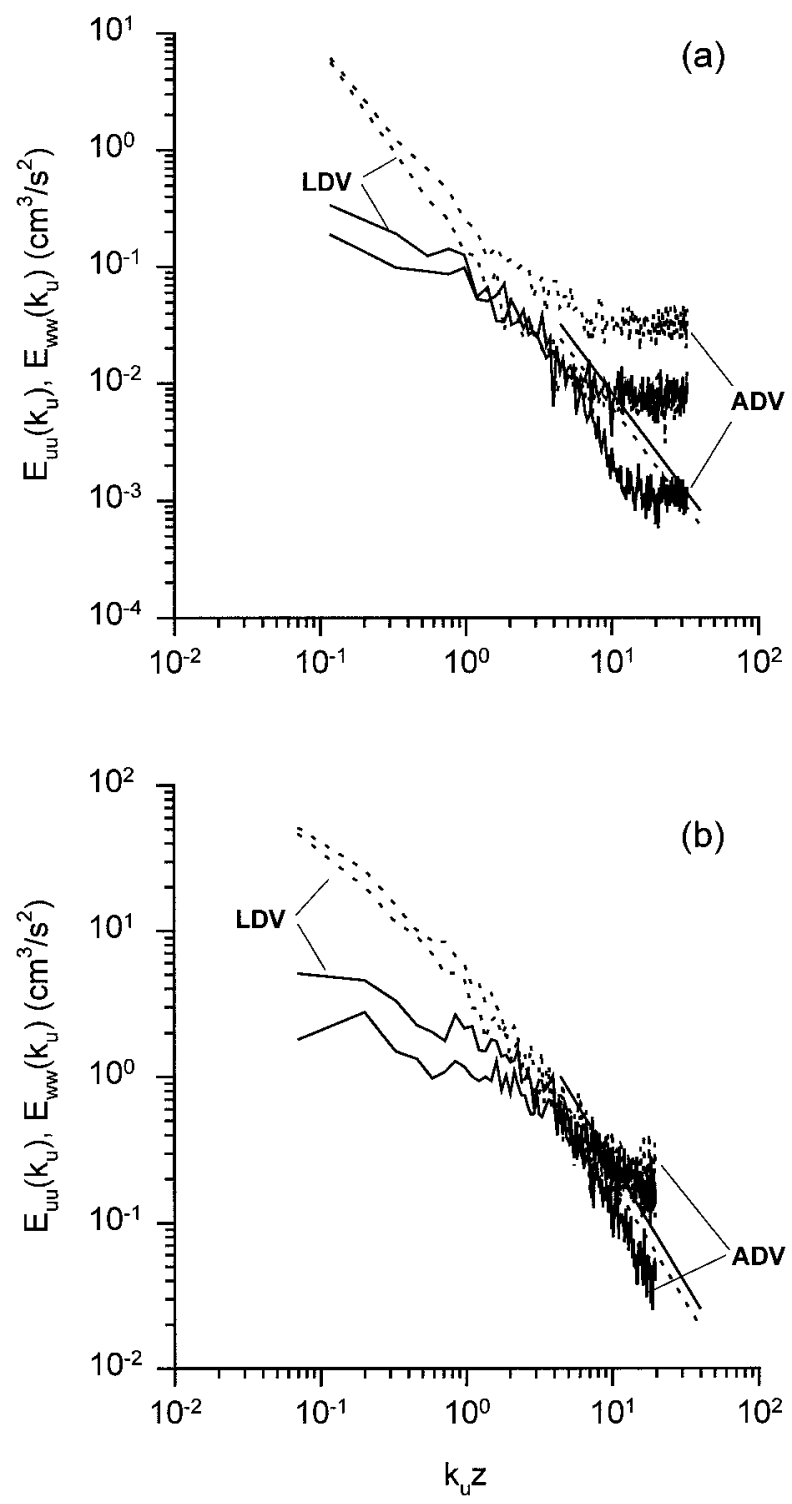

FIG. 7. Both ADV and LDV turbulence velocity spectra along the horizontal $\left[E_{\mathrm{uu}}\left(k_{u}\right)\right.$, solid lines] and vertical $\left[E_{\mathrm{ww}}\left(k_{u}\right)\right.$, dotted lines] for two typical runs with boundary Reynolds numbers $\left(u_{*} \kappa z / v\right) 56$ and 528 for diagrams (a) and (b), respectively. Flow conditions for the spectra shown. Panel (a): $h=25.5 \mathrm{~cm} ; z=3.1 \mathrm{~cm}, U=7.4 \mathrm{~cm}$ $\mathrm{s}^{-1}$, and $u_{*}=0.45 \mathrm{~cm} \mathrm{~s}^{-1}$. Panel (b): $h=25.0 \mathrm{~cm}, z=8.1 \mathrm{~cm}, U$ $=32.1 \mathrm{~cm}$, and $u_{*}=1.63 \mathrm{~cm} \mathrm{~s}^{-1}$. Shear velocities $u_{*}$ were derived using Eq. (21) with Reynolds stress $\left\langle u^{\prime} w^{\prime}\right\rangle$ values based on the ADVrecorded velocities. [Note: Straight lines represent Kolmogorov's turbulence spectra model, Eq. (27), for $k_{u} z>4.5$.]

during this experimental run were $h=25.5 \mathrm{~cm}, U=$ $7.4 \mathrm{~cm} \mathrm{~s}^{-1}$, and $u_{*}=0.45 \mathrm{~cm} \mathrm{~s}^{-1} .^{1}$ Despite the small Reynolds number $\left(u_{*} \kappa z / \nu=56\right)$ of the flow during this experiment, the calculated spectra are compared to Kol-

\footnotetext{
${ }^{1}$ The $u_{*}$ value used here was derived using Eq. (21) with the Reynolds stress $\left(\left\langle u^{\prime} w^{\prime}\right\rangle\right)$ calculated from the velocity data collected with the ADV.
} 
mogorov's turbulent spectra model (Tennekes and Lumley 1989). According to this model, the turbulence spectrum of the horizontal velocity $\left[E_{\mathrm{uu}}\left(k_{u}\right)\right]$ in the inertial subrange $\left(k_{u} \kappa z>1.8\right.$, i.e., $\left.k_{u} z>4.5\right)$ is given by

$$
E_{\mathrm{uu}}\left(k_{u}\right)=\frac{9}{55} \alpha \epsilon^{2 / 3} k_{u}^{-5 / 3},
$$

where $\alpha=1.5$, while the spectrum of the vertical component $\left[E_{\mathrm{ww}}\left(k_{u}\right)\right]$ is $(4 / 3) E_{\mathrm{uu}}\left(k_{u}\right)$.

The low energy conditions of the flow represented by the spectra shown in Fig. 7a offer insight into the limits of the instrument regarding instrument noise levels. The vertical component spectrum hits the noise floor $(0.001$ $\mathrm{cm}^{3} \mathrm{~s}^{-2}$ ) at $k_{u} z$ values just greater than 10 , while the horizontal component spectrum approaches the noise floor $\left(0.03 \mathrm{~cm}^{3} \mathrm{~s}^{2}\right)$ at smaller $k_{u} z$ values $(\simeq 8)$. The ratio of the spectral energy at the noise levels $\left(E_{\mathrm{uu}} / E_{\mathrm{ww}}=30\right)$ extracted from Fig. 7a agrees with the expected noise ratio (30.5) as derived from Eqs. (6) and (8) using the transformation matrix values shown in Eq. (4). This agreement confirms the validity of the above equations and the assumptions involved in their derivation. At normalized wavenumbers $\left(k_{u} z\right)$ greater than 4.5 (i.e., where the inertial dissipation range starts) and smaller than those that the spectra hit the noise floor, the horizontal velocity spectrum contains more energy than the vertical spectrum, which is the opposite of what is expected according to the Kolmogorov's model. The LDVmeasured spectra agrees with the vertical spectra of the ADV until it hits its noise floor. The energy of the vertical component of the LDV spectrum is slightly higher than the spectra of the horizontal component, as predicted by Kolmogorov's model.

The ADV and LDV spectra from an experimental run with higher energetic conditions $\left(U=32.1 \mathrm{~cm} \mathrm{~s}^{-1} ; u_{*}\right.$ $=1.63 \mathrm{~cm} \mathrm{~s}^{-1}$, see footnote 1) are shown in Fig. 7b. The measurement height was $8.1 \mathrm{~cm}$, while the mean water depth was $25.5 \mathrm{~cm}$. The Reynolds number for this run is 528 , which is higher than that of the example shown in Fig. 8a but still low in relation to the crudely estimated minimum value (2500) required for the development of the inertial subrange (Huntley 1988). In this example, the spectra of the ADV vertical component decays at high $k_{u} z$ values with a $-5 / 3$ rolloff all the way up to high $k_{u} z$ values. The horizontal component exhibits a similar decay up to $k_{u} z=10$ and then a sudden change in the slope is observed, indicating that the noise floor (approximately $0.12 \mathrm{~cm}^{3} \mathrm{~s}^{-2}$ ) is approached. The reversal in relative energy of the horizontal and vertical components observed in the example of Fig. 7a is present in Fig. 7b as well.

In both examples, the modeled Kolmogorov's spectra show higher energy levels than the measured ones, while the measured horizontal spectra contain more energy than the corresponding measured spectra of the vertical component of the flow. The origin of these discrepancies is explained below.
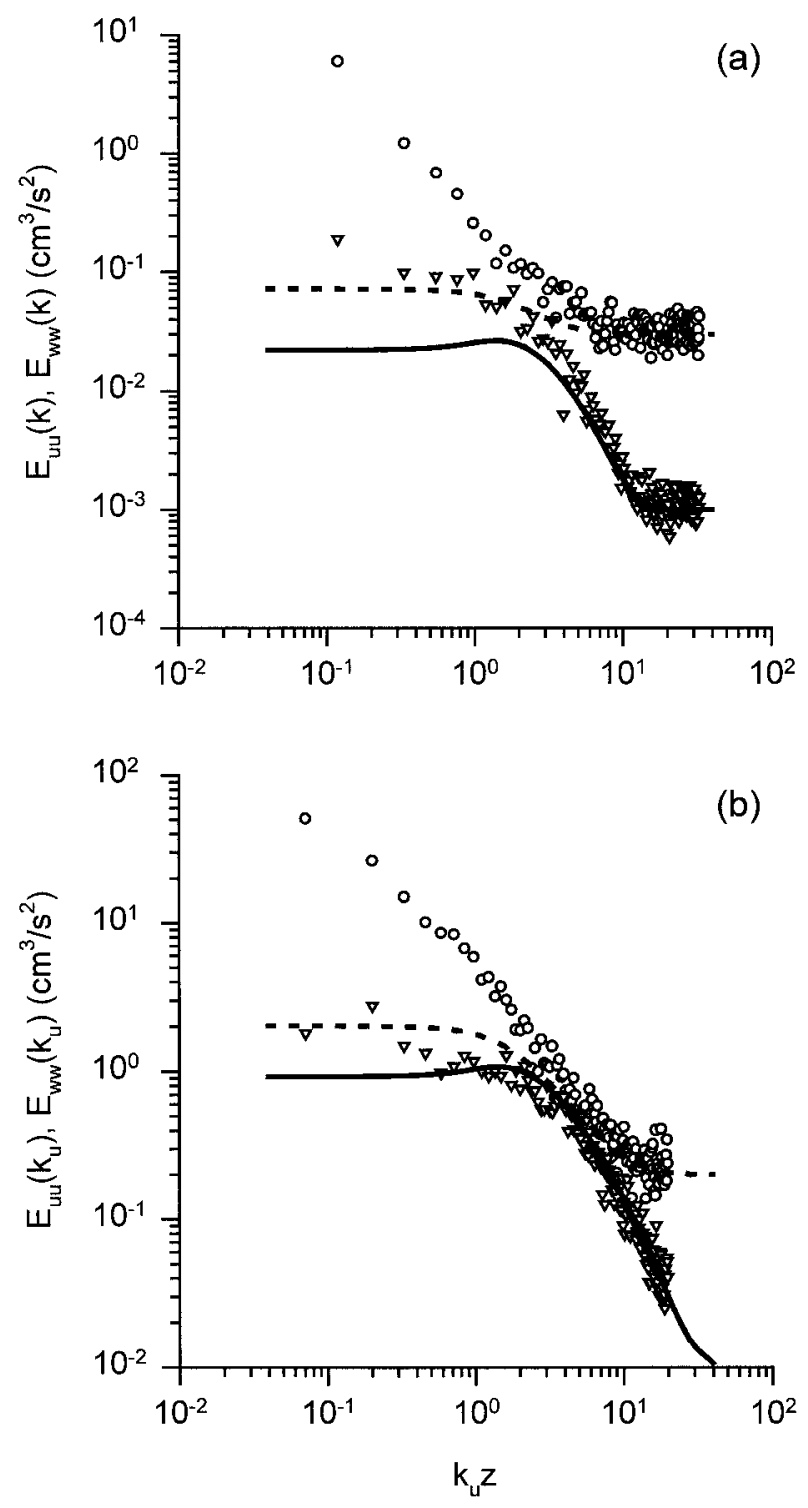

FIG. 8. ADV turbulence velocity spectra shown in Fig. 7 compared to Kolmogorov's turbulence spectra as modified to account for 1) viscous dissipation effects, 2) production effects, 3) attenuation due to spatial averaging within the sample volume, and 4) noise variance. \{Note: The triangles and solid lines represent turbulence spectra of vertical flow $\left[E_{\mathrm{ww}}\left(k_{u}\right)\right]$; circles and dashed lines denote alongstream turbulence spectra $\left.\left[E_{\text {uи }}\left(k_{u}\right)\right].\right\}$

In Fig. 8a, the same ADV spectra displayed in Fig. 7 are shown with the theoretical Kolmogorov's model modified to account for 1) viscous dissipation effects (Pao's form of spectrum); 2) production effects; 3) attenuation due to spatial averaging, corresponding to that of the ADV sample volume (see section 2a); and 4) contamination by measurement noise levels as observed at high wavenumbers in Fig. 7. The modified Kolmogorov's spectra shown in Fig. 8 were derived numeri- 
cally following George and Lumley's (1973) analysis, from the three-dimensional spectrum $E(k)$ :

$$
E_{\mathrm{uu}}\left(k_{u}\right)=\iint_{-\infty}^{+\infty} \frac{E(k)}{4 \pi k^{4}}\left(k^{2}-k_{u}^{2}\right) W(k) d k_{v} d k_{w}+\sigma_{\mathrm{uu}}^{2}
$$

and

$$
E_{\mathrm{ww}}\left(k_{u}\right)=\frac{1}{2} \iint_{-\infty}^{+\infty} \frac{E(k)}{4 \pi k^{4}}\left(k^{2}+k_{u}^{2}\right) W(k) d k_{v} d k_{w}+\sigma_{\mathrm{ww}}^{2},
$$

where

$$
\begin{aligned}
E(k)= & \left(\alpha \epsilon^{2 / 3} k^{-5 / 3}\right) \exp \left[-\frac{3}{2} \alpha(k \eta)^{4 / 3}\right] \\
& \times \exp \left[-\frac{3}{2} \pi \beta \alpha^{1 / 2}(k \kappa z)^{-4 / 3}\right]
\end{aligned}
$$

Here, $\eta$ is the Kolmogorov microscale defined by $\eta=$ $\left(\nu^{3} / \epsilon\right)^{1 / 4}$, where $\epsilon=\left(u_{*}^{3} / \kappa z\right)$ is the rate of dissipation of turbulent energy per unit mass; $\nu$ is the kinematic viscosity; $\beta=0.3$ [Tennekes and Lumley 1989, Eq. (8.4.10) with $l=\kappa z]$. The second and third factors in Eq. (30) account for the effect of viscous dissipation and production of turbulence, respectively. In the above equations $k$ represents the modulus of the three-dimensional wavenumber (i.e., $k^{2}=k_{u}^{2}+k_{v}^{2}+k_{w}^{2}$ ). The noise levels for the example shown in Fig. 8a were derived from the spectra shown in Fig. 7a. The noise level for the $u$ component of the example shown in Fig. $7 \mathrm{~b}$ was calculated from the spectra, while for the $w$ component of the relationship $\sigma_{\mathrm{uu}}^{2}=30.5 \sigma_{\mathrm{ww}}^{2}$ [based on Eqs. (6) and (8)] was used. The modified Kolmogorov's spectra resemble the ADV-measured spectra with the energy along the vertical component being smaller than that of the horizontal. It is noteworthy that the turbulence spectra of the turbulent component exhibit a much better agreement than the spectra of the horizontal flow component. The latter seem not to be affected by the production effects. This might be because no isotropic turbulence conditions existed during the experimental conditions and/or due to the existence of low-frequency oscillations.

\section{f. Noise analysis}

The results presented so far have displayed that the ADV is capable of measuring accurate mean flows, Reynolds stresses, and vertical turbulence intensities. Noise affecting the quality of the signal is cancelled in the mean values, whereas for the Reynolds stress and the vertical variance it is reduced because of the sensor's geometrical characteristics. However, the noise degrades the signal in the horizontal component.

Accurate estimates of the noise levels could be useful for the interpretation of data collected using the ADV and even for the correction of the spectra for an accurate study of the turbulence structure. However, such an attempt requires separation of the noise and real signal components. In the present work, the total noise variance $\sigma_{t}^{2}$ for measurements obtained during experiments $\mathrm{A}$ and $\mathrm{B}$ was calculated by two independent methods. The first method, spectral analysis, calculates the noise as the variance included in the tail of the spectrum (frequency range $11.5-12.5 \mathrm{~Hz}$, chosen so that there are 10 estimates for the calculation of a statistically significant average). The total noise estimate was derived by integrating over the whole range of frequencies, assuming white noise. The second method uses the results of the ground truthing procedure described in section $3 \mathrm{c}$. Both methods, however, have disadvantages. Integrating over the high frequencies of the spectra might be considered an accurate method for runs with very low flow energy. At highly energetic flows, the turbulent signal extends to high frequencies so that the variance at the tail of the spectra consists of both signal and noise components. On the other hand, although the ground truthing method seems to be a robust approach, it could overestimate noise variances. Whatever is predicted as noise $\left(\sigma_{t}^{2}\right)$ is not necessarily due to electronics, Doppler broadening, or shear within the sample volume. Errors due to misalignment of the ADV and LDV sensors or due to sensor oscillations (vibration) at different directions and/or different amplitudes will be classified as noise variance with the ground truthing technique. Consequently, both spectral and ground truthing techniques are expected to overestimate the total noise variances. Noise estimates derived from both techniques are compared with each other and with estimations based on the theoretical approximations of noise described in section 2b (Fig. 9).

Total noise estimates from the spectra and ground truth techniques are compared in Fig. 9a. Both of them exhibit a good correlation with a squared correlation coefficient of 0.851 . However, the noise from the ground truthing method is approximately 3.5 times greater than that obtained from the tail of the spectra. This might be the noise variance that exists at lower frequencies (i.e., vibration) and has not been included in the estimations derived from the high frequencies (tail) of the spectra.

Comparison of the theory, as described in section $2 b$ [Eqs. (9)-(14)], with the flow-related noise from the ground truth technique (section $3 \mathrm{c}$ ) and the estimations derived using the tail of the measured spectra are shown in Figs. 9b and 9c, respectively. The latter estimations are in better agreement with the theory $\left(r^{2}=0.570\right)$ than the former $\left(r^{2}=0.388\right)$ with the magnitude of the spectral estimations being closer to the theoretical ones than that obtained with the ground truthing technique (Fig. 9b).

The difference between the total and the instrument noise variance defined above is equal to noise variance terms, which depend on the actual flow characteristics 

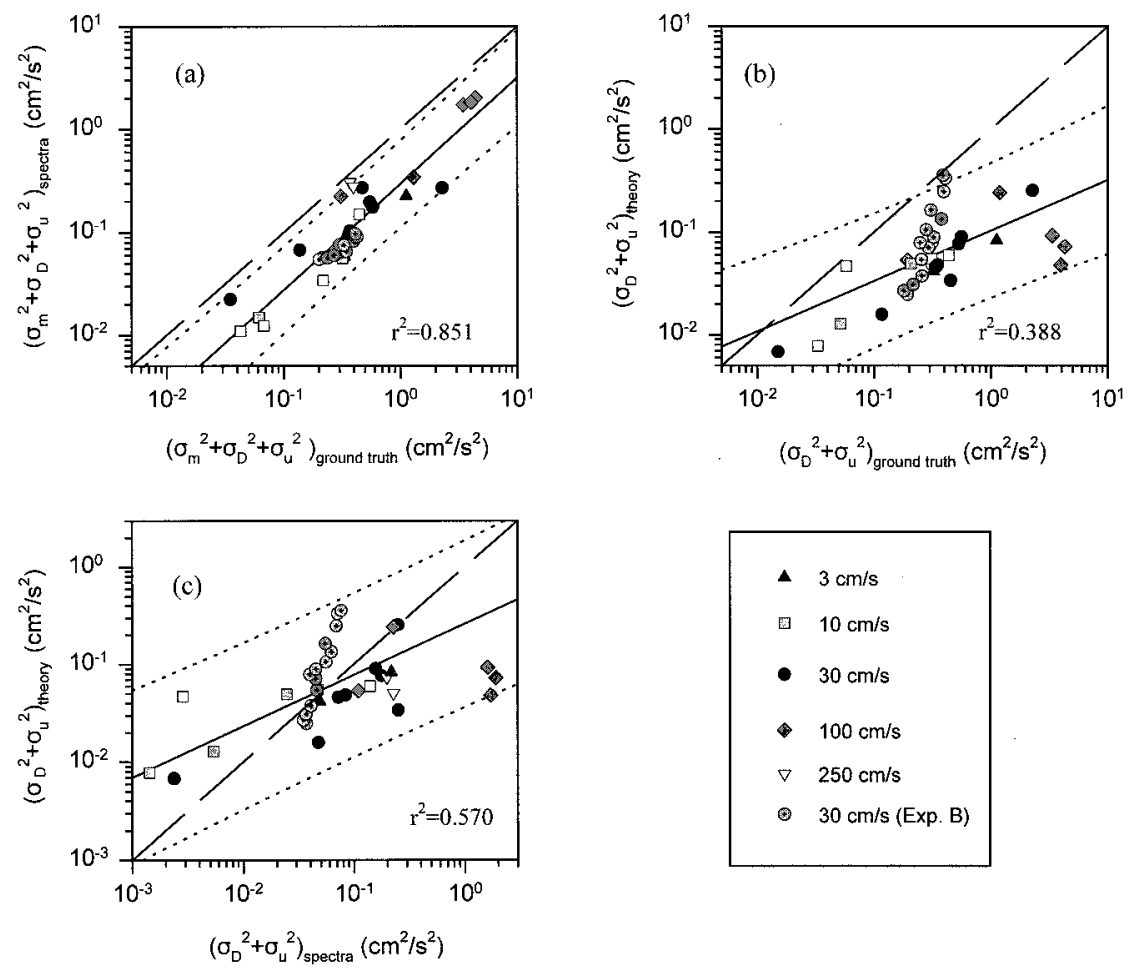

FIG. 9. Intercomparison of total noise estimated from the tail of the spectra and from the ground truthing analysis and (a) of flow-related noise estimated using the above methods and the theoretical predictions based on Eqs. (10)-(16) [diagrams (b) and (c)]. The dashed line is the 1:1 line, while the dotted line denotes the $95 \%$ confidence interval of the best-fit regression analysis (solid line).

within the sample volume (Doppler noise $\sigma_{D}^{2}$ and velocity gradient noise $\sigma_{u}^{2}$, respectively). The turbulence term [Eq. (14)] of the Doppler noise and the term associated with shear within the sample volume [Eq. (16)] are the most important noise sources in boundary layer measurements (Figs. 3c and 3d). Both quantities that are functions of shear velocity and their sum $\left(\sigma_{D}^{2}+\right.$ $\sigma_{u}^{2}$, flow-related noise) would be related to the turbulence dissipation factor $\epsilon$ in a power of $2 / 3$. This simplification ignores other terms, which include the ratio of $d / z$, and thus are more accurate for measurements obtained at elevations a few times greater than the sample volume size. The flow-related noise terms as expressed by the theory through Eqs. (11)-(17) are empirically parameterized into a single equation that associates this noise term to turbulence dissipation:

TABLE 5. Results of regression analysis shown in Fig. 9. The values of the coefficients $A$ and $B$ are shown together with their $95 \%$ confidence intervals.

\begin{tabular}{lc}
\hline \hline$\left(\sigma_{D}^{2}+\sigma_{u}^{2}\right)=10^{A} \epsilon^{2 / 3}$ & $A$ \\
\hline Theory vs $\epsilon$ & $-0.971 \pm 0.061$ \\
Spectra tail vs $\epsilon$ & $-0.910 \pm 0.172$ \\
Ground truth vs $\epsilon$ & $-0.231 \pm 0.116$ \\
\hline
\end{tabular}

$$
\sigma_{D}^{2}+\sigma_{u}^{2}=10^{A} \epsilon^{2 / 3} .
$$

It should be noted that the above equation is dimensionally inconsistent and that the coefficient $A$ depends on the size of the sample volume, operating frequency, duration of acoustic pulse, and other sensor-dependent characteristics. Thus, the validity of Eq. (31) is limited to the sensor type used in the present study.

The coefficient $A$ was calculated from the experimental data for the theoretical parameterization, the spectral estimates, and the results of the ground truth analysis. The magnitude and significance level of the calculated values are shown in Table 5. A good agreement is found between the value based on the parameterization of the theory and of that based on the tail of the spectra, while the $A$ value based on the ground truth technique is almost four times greater. This disagreement between the theoretical and ground truth derived $A$ values is due to the fact that the ground truth method overestimates the noise by including such errors as sensor misalignment and/or vibration (see discussion above). The above results indicate that Eq. (29), with $A=-0.97$, constitutes a good first-order approximation for the evaluation of flow-induced noise in the ADV measurements. 


\section{Conclusions}

Evaluation of the ADV sensor was undertaken in the laboratory under steady flow conditions. The analysis of the data collected in conjunction with an LDV sensor showed the following.

- Mean flows measured with the ADV agree within $1 \%$ with those measured by the LDV (rms error $5.6 \mathrm{~mm}$ $\mathrm{s}^{-1}$ ). This difference is attributed to uncertainties in vertically aligning the two sensors. The high accuracy and the inherent property of zero-drift free velocity measurements make the ADV suitable for accurate measurements of mean flow even at positions close to the boundary $(z \simeq 0.75 \mathrm{~cm})$.

- ADV-measured Reynolds stress values were underestimated by only $1 \%$, even without any correction for noise. The accuracy of these measurements was also confirmed by the ability of the sensor to describe the vertical variation of the Reynolds stress according to existing models for open channel flow.

- Turbulence intensity of the vertical component can be resolved accurately by the sensor, while the intensity of the downstream components suffers from a high noise term that is an inescapable feature of the geometry of the ADV.

- Good agreement was found between bottom shear velocities $u_{*}$ calculated using the Reynolds stress and logarithmic profile methods.

- Spectral estimations of the horizontal and vertical flow components agree with theoretical spectral estimations based on Kolmogorov's model if correction is applied for 1) viscous dissipation effects, 2) production effects, 3) spatial averaging of the sample volume, and 4) measurement noise variance.

- The noise variance along the measuring beam of the ADV signal consists of an electronics-related component (ability of the sensor to resolve the phase of a pair of pulses) and a flow-related component. The latter appear to dominate at high flow rates, and it has been shown to be primarily a function of the turbulence dissipation factor. Noise variances estimated using the spectra of the signal were smaller than noise variances derived using the ground truthing technique but in good agreement with the theory. The latter is suggested as a first-order approximation for the prediction of noise levels.

Acknowledgments. D. G. Aubrey is thanked for his support and interest in the progress of the project. J. Sisson's assistance during the experiments was invaluable. The authors also are indebted to E. A. Terray and A. Hayes for the fruitful discussions during the course of this investigation. Financial support for G. Voulgaris was provided by the Woods Hole Oceanographic Institution in the form of a Postdoctoral Scholar Award funded by the Mellon Foundation and for J. Trowbridge by the Office of Naval Research (ONR), Coastal Sciences.

\section{REFERENCES}

Agrawal, Y. C., and C. J. Belting, 1988: Laser velocimetry for benthic sediment transport. Deep-Sea Res., 35, 1047-1067.

- and D. G. Aubrey, 1992: Velocity observations above a rippled bed using laser Doppler velocimetry. J. Geophys. Res., 97, 20 249-20 259.

Anderson, S., and A. Lohrmann, 1995: Open water test of the SonTek Acoustic Doppler Velocimeter. Proc. IEEE Fifth Working Conf. on Current Measurements, St. Petersburg, FL, IEEE Oceanic Engineering Society, 188-192.

Blevins, R. D., 1977: Flow Induced Vibration. Van Nostrand Reinhold, $363 \mathrm{pp}$.

Blinco, P. H., and E. Partheniades, 1971: Turbulence characteristics in free surface flows over smooth and rough boundaries. J. Hydraul. Res., 9, 43-69.

Brumley, B. H., R. G. Cabrera, K. L. Deines, and E. A. Terray, 1991: Performance of a broad-band acoustic Doppler current profiler. IEEE J. Oceanic Eng., 16, 402-407.

Buchhave, P., W. K. George, and J. L. Lumley, 1979: The measurement of turbulence with the laser Doppler velocimeter. Аnпи. Rev. Fluid Mech., 11, 443-503.

Butman, C. A., and R. J. Chapman, 1989: The 17-meter flume at the Coastal Research Laboratory. Part I: Description and user's manual. Woods Hole Oceanographic Institution Tech. Rep. WHOI89-10 (CRC-89-2), 31 pp. [Available from Document Library, Woods Hole Oceanographic Institution, Woods Hole, MA 02543.]

Cabrera, R., K. Deines, B. Brumley, and E. Terray, 1987: Development of a practical coherent acoustic Doppler current profiler. Proc. Oceans '87, Halifax, NS, Canada, IEEE Oceanic Engineering Society, 93-97.

Cacchione, D. A., and D. E. Drake, 1979: A new instrument system to investigate sediment dynamics on continental shelves. Mar. Geol., 30, 299-312.

Clay, C. S., and H. Medwin, 1977: Acoustical Oceanography: Principles and Applications. Wiley, $544 \mathrm{pp}$.

George, W. K., and J. L. Lumley, 1973: The laser-Doppler velocimeter and its application to the measurement of turbulence. J. Fluid Mech., 60, 321-362.

Grass, A. J., 1971: Structural features of turbulent flow over smooth and rough boundaries. J. Fluid Mech., 65, 439-459.

Guza, R. T., and E. B. Thornton, 1980: Local and shoaled comparisons of sea surface elevations, pressures and velocities. J. Geophys. Res., 85, 1524-1530.

Huntley, D. A., 1988: A modified inertial dissipation method for estimating seabed stresses at low Reynolds numbers, with application to wave/current boundary layer measurements. J. Phys. Oceanogr., 18, 339-346.

Kraus, N. C., A. Lohrmann, and R. Cabrera, 1994: New acoustic meter for measuring 3D laboratory flows. J. Hydraul. Eng., 120, 406-412.

Lhermitte, R., and U. Lemmin, 1994: Open-channel flow and turbulence measurement by high-resolution Doppler sonar. J. Atmos. Oceanic Technol., 11, 1295-1308.

Lohrmann, A., R. Cabrera, and N. C. Kraus, 1994: Acoustic-Doppler velocimeter (ADV) for laboratory use. Proc. Conf. on Fundamentals and Advancements in Hydraulic Measurements and Experimentation, Buffalo, NY, American Society of Civil Engineers, 351-365.

$\_,-$, G. Gelfenbaum, and J. Haines, 1995: Direct measurements of Reynolds stress with an acoustic Doppler velocimeter. Proc. IEEE Fifth Conf. on Current Measurements, St. Petersburg, FL, IEEE Oceanic Engineering Society, 205-210.

Miller, K. S., and M. M. Rochwarger, 1972: A covariance approach to spectral moment estimation. IEEE Trans. Inform. Theory, IT18, 588-596.

Nakagawa, H., and I. Nezu, 1981: Predictions of the contributions to the Reynolds stress from the bursting events in open channel flows. J. Fluid Mech., 104, 1-43. 
Nezu, I., and W. Rodi, 1986: Open channel flow measurements with a laser Doppler anemometer. J. Hydraul. Eng., 112, 335-355.

Smith, J. D., and S. R. McLean, 1977: Spatially averaged flow over a wavy surface. J. Geophys. Res., 82, 1735-1746.

SonTek, 1995: ADV operation manual, version 1.0. 30 pp. [Available from SonTek, 6837 Nancy Ridge Drive, Suite A, San Diego, CA 92121.]

Tennekes, H., and J. L. Lumley, 1989: A First Course in Turbulence. The MIT Press, 300 pp.

Trowbridge, J. H., and Y. C. Agrawal, 1995: Glimpses of a wave boundary layer. J. Geophys. Res., 100, 20729-20743.

- W. R. Geyer, C. A. Butman, and R. J. Chapman, 1989: The 17-meter flume at the Coastal Research Laboratory. Part II: Flow characteristics. Woods Hole Oceanographic Institution Tech.
Rep. WHOI-89-11 (CRC-89-3), 37 pp. [Available from Document Library, Woods Hole Oceanographic Institution, Woods Hole, MA 02543.]

Voulgaris, G., M. Wilkin, and M. B. Collins, 1995: The in-situ acoustic measurements of shingle movement under waves and currents: Instrument (TOSCA) development and preliminary results. Con. Shelf Res., 15, 1195-1211.

Williams, A. J., III, J. S. Tochko, R. L. Koehler, T. F. Gross, W. D. Grand, and C. V. R. Dunn, 1987: Measurement of turbulence with an acoustic current meter array in the oceanic bottom boundary layer. J. Atmos. Oceanic Technol., 4, 312-327.

Zedel, L., A. E. Hay, and A. Lohrmann, 1996: Performance of a single beam pulse-to-pulse coherent Doppler profiler. IEEE J. Ocean Eng., 21, 290-299. 